\title{
Investigation of the role of pedestal pressure and collisionality on type-I ELM divertor heat loads in DIII-D
}

M. Knolker(1), A. Bortolon(1), G.P. Canal( ${ }^{(2)}$, T.E. Evans ${ }^{(2)}$, H. Zohm ${ }^{(3)}$, T. Abrams ${ }^{(2)}$,R.J. Buttery ${ }^{(2)}$, E.M. Davis $^{(4)}$, R.J. Groebner ${ }^{(2)}$, E. Hollmann ${ }^{(5)}$, M.E. Fenstermacher ${ }^{(6)}$, C. Lasnier $^{(6)}$, A.W. Leonard ${ }^{(2)}$, R.A.

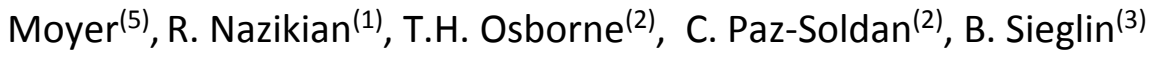

(1)Princeton Plasma Physics Laboratory, Princeton, New Jersey

${ }^{(2)}$ General Atomics, San Diego, California

${ }^{(3)}$ Max Planck Institut fuer Plasmaphysik, Garching, Germany

${ }^{(4)}$ Massachusetts Institute of Technology, Boston, Massachusetts

${ }^{(5)}$ University of California at San Diego, La Jolla, California

${ }^{(6)}$ Lawrence Livermore National Laboratory, Livermore, California

ABSTRACT

A non-dimensional collisionality scan conducted on DIII-D confirms a model for ELM energy densities recently put forward by Eich [1], but also reveals key effects that may explain the large scatter typically observed about the scaling. Electron Cyclotron Heating 
$(\mathrm{ECH})$ close to the plasma edge was used to raise electron temperatures at the pedestal top and lower collisionality to ITER level, while the power of Neutral Beam Injection (NBI) was decreased during discharges to operate closer to the L-H transition threshold. The scan reveals no explicit pedestal pressure dependence of the ELM energy densities. While collisionality does not play a decisive role, the ratio of heating power to the power over the $\mathrm{L}-\mathrm{H}$-threshold is identified as parameter determining the agreement with the model, with discharges marginally above the threshold showing the largest scatter in the database and exceeding the predicted ELM energy up to twofold. Operation close to the $\mathrm{L}-\mathrm{H}$-threshold is accompanied by low ELM frequency and large ELM heat loads. Using linear stability calculations, ELM energy densities are shown scale inversely with to the most unstable linear mode number before the ELM crash. There are indications that the scatter in the data when compared with the Eich model prediction is caused by including only a limited set out of all quantities considered by linear stability analysis. While further ELM studies near the LH threshold are of great priority, the overall agreement of DIII-D with the Eich model recommends its use in extrapolations towards ITER.

\section{ACKNOWLEDGEMENTS}

*This material is based upon work supported by the U.S. Department of Energy, Office of Science, Office of Fusion Energy Sciences, under Award Numbers DE-FC02-04ER54698, DE-FG0207ER54917 and DE-FG02-05ER54809. This research used resources of the DIII-D National Fusion Facility, which is a DOE Office of Science User Facility. We gratefully acknowledge the support of the DIII-D Team for tokamak, auxiliary heating, and diagnostic systems operation. Part of the data analysis was performed using the OMFIT integrated modeling framework [2].

DISCLAIMER 
This report was prepared as an account of work sponsored by an agency of the United States Government. Neither the United States Government nor any agency thereof, nor any of their employees, makes any warranty, express or implied, or assumes any legal liability or responsibility for the accuracy, completeness, or usefulness of any information, apparatus, product, or process disclosed, or represents that its use would not infringe privately owned rights. Reference herein to any specific commercial product, process, or service by trade name, trademark, manufacturer, or otherwise, does not necessarily constitute or imply its endorsement, recommendation, or favoring by the United States Government or any agency thereof. The views and opinions of authors expressed herein do not necessarily state or reflect those of the United States Government or any agency thereof. 


\section{Introduction}

Edge-localized-modes (ELMs) are a ubiquitous standard H-mode phenomenon characterized through the collapse of the edge pressure gradient and bootstrap current in a repetitive cycle. While ELMs facilitate impurity transport and thereby prevent a radiative plasma collapse [3], they pose a threat on next-step devices, by causing erosion, melting and recrystallization in the divertor $[4,5]$. In recent material studies, tungsten samples, the material foreseen for the ITER divertor, were subjected to transient thermal loads representative of the heat pulses caused by ELMs in future power plants [6]. The experiments revealed material limits below the expected loads for ITER, implying that critical damage will be reached after one discharge with uncontrolled ELMs. Thus, ELM control strategies have been developed, as naturally ELM free regimes such as QH-mode [7], ELM suppression using resonant magnetic perturbations (RMP) [8], or ELM mitigation with impurity seeding [9].

The declared minimal goal of the design commissions for ITER was to drastically reduce the size of large type-I ELMs by a factor of at least 20 to avoid divertor damage while maintaining the impurity transport $[5,10]$. By employing an ion orbit model and accounting for the exact divertor geometry, the latest simulations indicate that the tile edges are most sensitive to ELM heat loads [11]. Considering these results, ITER's ELM energy density should be limited to $0.15 \mathrm{MJ} / \mathrm{m}^{2}$ (down from $0.5 \mathrm{MJ} / \mathrm{m}^{2}$ ) to prevent edge melting. Thus, uncontrolled ELMs present a major operational constraint for ITER.

The ELM mitigation requirements are based on extrapolations which depend on models. Thus, it is vital to understand the scaling of ELM heat loads with plasma parameters to design a benign scenario and define the requirements for mitigation. Based on experimental findings on ASDEX Upgrade (AUG) and JET a model was put forward proposing that parallel ELM energy densities scale with pedestal pressure, from here on referred to as the Eich model [1]. Most of the data which the model is based on was taken in plasmas with collisionalities above ITER expected values due to operational limits associated with metal walls. The Eich model should be contrasted with Loarte's previous extrapolations that propose ELM size scales with electron collisionality in the pedestal [12]. For the low collisionality edge in the active ITER phase, Loarte's approach predicts 
heat loads up to two times larger than those predicted by the Eich model. To test the validity of the Eich model at ITER-relevant pedestal collisionality, experiments were conducted on the DIIID tokamak to measure ELM heat loads and their deposition patterns at the divertor's inner and outer strike points. Data from previous standard H-mode experiments on DIII-D with attached divertor legs, IR monitoring and no applied ELM control is also considered, in order to increase the parameter range of the analysis.

The remainder of the paper is structured as follows: In section 2 the Eich Model for ELM energy densities is introduced and compared to established scaling laws. The experimental setup and utilized diagnostics are described in section 3. In Section 4 the test results of the Eich model on DIII-D are presented, followed by an investigation of potential hidden variables in the scaling. Among the potential hidden variables is the proximity to the LH-threshold, which is discussed in detail in section 5. Section 6 evaluates the degree to which linear-stability analysis provides information about (the nonlinearly driven) ELM energy densities. A summary of the key results and a discussion of necessary future steps is provided in section 7. 


\section{Eich Model}

The Eich model (EM) provides predictions for ELM heat loads in existing devices and allows extrapolation to ITER. The model was recently introduced in [1], where its predictions are compared against a multi-machine dataset, including measurements from MAST, AUG, and JET. In this section the main aspects of the model are outlined. The model characterizes ELMs in terms of a single quantity, the peak parallel ELM energy density $\varepsilon_{\|}$. Other relevant quantities such as divertor peak heat flux, heat flux width, and deposited energy, are to be derived a-posteriori, based on the properties of the magnetic equilibrium and knowledge of ELM dynamics. The peak parallel ELM energy density $\varepsilon_{\|, \text {peak }}$ is computed from the target ELM energy density $\varepsilon_{\text {tar }}$, defined as the spatial maximum of the time-integrated heat flux $q(s, t)$

$$
\varepsilon_{\text {tar }}(s)=\int_{\tau_{E L M}} q(s, t) d t
$$

Where $s$ is the radial coordinate along the tile surface, $\mathrm{t}$ is the time and $\tau_{E L M}$ is the ELM duration. This quantity is generally measured by infrared thermography. In order to allow comparisons between different machines or divertor configurations, the target ELM energy density is projected onto magnetic field lines, to obtain the corresponding parallel ELM energy density $\varepsilon_{\|}(s)$. The peak parallel energy density is the spatial maximum of $\varepsilon_{\|}$:

$$
\varepsilon_{\|, p e a k}=\max \left(\frac{\varepsilon_{\text {tar }}(s)}{\sin \gamma(s)}\right)=\max \left(\frac{\int_{\tau_{E L M}} q(s, t) d t}{\sin \gamma(s)}\right),
$$

where $\gamma$ represents the incidence angle of the magnetic field lines with respect to the tile surface. To simplify notation, in the following $\varepsilon_{\|}$will be used for the peak parallel ELM energy density. The standard unit for $\varepsilon_{\|}$is $\mathrm{MJ} / \mathrm{m}^{2}$. Typically, the location of the maximum is close to the strike point for narrow heat flux profiles, but it can be significantly different depending on the magnetic-field configuration, in particular for broader heat flux profiles. As indicated in [4], $\varepsilon_{\|}$represents an appropriate quantity for ELM heat load extrapolation when considering the dynamic of melting and erosion. 
In fact, the zones that deviate from the nominal monolithic divertor surface (e.g. tile edges) are most susceptible to melting and recrystallization. These zones are small, so that for defining heat load-limits it is enough to compare the peak ELM energy density with the material limit at the zone. In contrast, calculations as in [3] are based on measurements of heat flux width-averaged ELM loads and will, for short-term exposures, be less restrictive than projections based solely on the peak value. The measurements of heat flux width remain important for estimating average lifetime material strain [13].

Note, that ELM dynamics are lost in the time integration. However, as inter-machine comparisons have shown that type-I ELMs have similar temporal heat flux profiles in the divertor [10], the ELM energy density is a suitable quantity for heat load projections. As a practical caveat, when computing $\varepsilon_{\|}$from experimental ELM heat flux profiles, it is important to define a standard procedure for determining the integration time-interval. Furthermore, comparisons are more accurate if only ELMs with similar dynamics are included. For example, exotic compound ELMs with a low peak heat flux but much longer duration (compared to a standard type I ELM) result in high $\varepsilon_{\|}$values, which do not necessarily imply the same hazard, due to their lack of a comparable sudden rise in the peak heat flux [14]. In contrast to a pure peak heat flux comparison, $\varepsilon_{\|}$is not affected by the choice of the divertor surface parameter in the IR thermography, as explained in the appendix.

The Eich model assumes a direct flux tube connection between the pedestal top and the divertor during an ELM, such that, the width of the peeled-off pedestal layer determines the deposition width in the divertor. This assumption produces an optimistic scaling for the heat flux broadening during an ELM. Based on these assumptions, the Eich model concludes that the peak ELM energy density scales as

$$
\varepsilon_{\|, E i c h}=6 \pi \cdot p_{e, p e d} \cdot a_{p o l} \cdot \frac{B_{T}}{B_{p}},
$$

where $p_{e, p e d}$ is the electron pressure at the pedestal top, $a_{p o l}$ is the minor radius of the plasma (corrected for elongation), and $B_{T}$ and $B_{p}$ are the toroidal and poloidal magnetic fields at the outboard midplane, respectively. Measurements of $\varepsilon_{\|}$in MAST, AUG and JET (the majority on the outer strike point), when compared with model predictions, were found to lie between $\varepsilon_{\|, \text {Eich }}$ 
and $3 \mathrm{x} \varepsilon_{\|, \text {Eich }}[1]$. While the Eich model was developed to explain heat loads in the outer divertor, measurements of the inner divertor also indicate agreement [1].

It is interesting to compare the predictions for ITER with the conventional approach to ELM heatload extrapolation[5]. For the non-active phase of ITER, the model predicts $\varepsilon_{\|}=2.5 \mathrm{MJ} / \mathrm{m}^{2}\left(\varepsilon_{t a r}=\right.$ $\left.0.13 \mathrm{MJ} / \mathrm{m}^{2}\right)$, for the active phase $10 \mathrm{MJ} / \mathrm{m}^{2}\left(\varepsilon_{\text {tar }}=0.5 \mathrm{MJ} / \mathrm{m}^{2}\right)$. The Loarte approach is based on the empirical scalings for plasma energy lost during an ELM, $W_{E L M}$, the wetted area and the experimental evidence that plasma energy losses during type-I ELMs are inversely related to the electron pedestal collisionality $v_{e}^{*}$ [15]. For ITER, the predicted $v_{e}^{*} \sim 0.06-0.1$ entails ELM sizes of up to $20 \%$ of the pedestal energy. In the non-active phase, a pedestal top temperature of 2.35 $\mathrm{keV}$ is predicted at a density of $4 \cdot 10^{19} \frac{1}{\mathrm{~m}^{3}}$ (active phase: $4.7 \mathrm{keV}, 8 \cdot 10^{19} \frac{1}{\mathrm{~m}^{3}}$ ). Approximating the pedestal energy with $T_{\text {e,ped }}+T_{i, p e d}=2 \cdot 2.35 \mathrm{keV}$ as

$$
W_{\text {ped }}=\frac{3}{2} \cdot n_{e, p e d} \cdot\left(T_{e, p e d}+T_{i, p e d}\right) \cdot V_{\text {plasma }}
$$

this results in $W_{E L M} \sim 4 M J$ for uncontrolled type-I ELMs. Assuming that during ELMs $60 \%$ of $W_{E L M}$ is transported to the divertor with a distribution of 2:1 in favor of the inner divertor $[16,17]$, 1.6 MJ will be deposited on the inner and $0.8 \mathrm{MJ}$ on the outer. Considering the result of empirical studies revealing that the SOL heat flux width $\lambda_{q}$ varies inversely with the plasma current [18], one obtains a wetted area of $1.6 \mathrm{~m}^{2}$ on the inner and $2.6 \mathrm{~m}^{2}$ on the outer divertor in the worstcase, no broadening scenario for the non-active phase $[19,20]$. Now, the target ELM energy densities can be determined to be $\varepsilon_{\text {tar,Loarte }}=1.10 \mathrm{MJ} / \mathrm{m}^{2}$ on the inner and $\varepsilon_{\text {tar,Loarte }}=0.32$ $\mathrm{MJ} / \mathrm{m}^{2}$ on the outer. The estimate for the active case follows on similar arguments. An overview and comparison of the two models is shown in table 1, where all values are target ELM energy densities in $\mathrm{MJ} / \mathrm{m}^{2}$ for inner and outer divertor.

In the table, Eich's model is listed with the lower and the threefold upper limits, whereas for Loarte's model, extrapolations are shown for the no broadening and threefold broadening cases. Since the Eich model is technically only valid for the outer divertor, the estimates for the inner divertor are calculated by multiplying the outer divertor estimates by the same in-out ratio as used in the Loarte extrapolations. Note that many of the lower estimates shown are well above 
the $0.15 \mathrm{MJ} / \mathrm{m}^{2}$ upper limit established in the latest divertor tolerance simulation [11]. The substantial differences between the models result from the different scaling of respective relevant pedestal quantities:

- In the Eich model, the ELM energy is solely determined by pedestal pressure $p_{e, p e d}=n_{e, p e d} \cdot T_{e, p e d}$. It assumes favorable decrease of the peeled off layer width during ELMs with plasma current resulting in $\varepsilon_{\|, E i c h} \sim \frac{1}{B_{p}}$, resulting in considerably lower heat loads in the active phase of ITER, while the results are comparable in the non-active phase.

- In Loarte's approach, the relevant quantity is the pedestal collisionality $v_{e}^{*} \sim \frac{n_{e, p e d}}{T_{e, p e d}^{2}}$, which determines the ELM size as a fraction of the pedestal energy. Due to heat flux width reduction and flux expansion the scaling with plasma current is quadratic $\varepsilon_{\|, \text {Loarte }} \sim \frac{1}{B_{p}{ }^{2}}$.

These differences in projections for ITER will be exacerbated in predictions for fusion power plants, where low collisionality will be required for sustainment of high bootstrap fractions characteristic of steady state operation [21]. 


\section{Experimental Setup and Diagnostics}

\section{Discharge scenario}

The experiment reported in this paper consisted of lower single null $\mathrm{H}$-mode plasmas on the DIII$D$ tokamak $(R=1.7 \mathrm{~m}, \mathrm{a}=0.6 \mathrm{~m})$ with $\mathrm{NBI}$ power between $\mathrm{P}_{\mathrm{NB}}=2-5 \mathrm{MW}, \beta_{N}=1.5-2.2$, and an edge safety factor $q_{95}=4.0-4.4$. $E C H$ power was injected near the plasma edge, resulting in density pump-out and increase of pedestal temperature, both effects that contribute to reduce collisionality. Up to $3.5 \mathrm{MW}$ of ECH power was deposited at $\rho=0.70-0.92$, close to the pedestal top, where pedestal electron temperatures in excess of $T_{e, p e d}=2 \mathrm{keV}$ have been achieved. A magnetic configuration with a lower triangularity of $0.74-0.78$, an upper triangularity of $0.33-0.39$ and an elongation of $1.77-1.80$ (figure 1) was chosen, close to the so-called ITER similar shape (ISS). During the experiment both the inner and outer strike points were monitored simultaneously with the fast infrared television camera (IRTV). In order to extend range of collisionality explored, the magnetic field was varied on a discharge by discharge case over a range $\mathrm{Bt}=1.6 \mathrm{~T}-2.15 \mathrm{~T}$.

An experimental goal was to execute a non-dimensional scan, i.e. keeping the dimensionless parameters $q_{95}$ and $\beta_{N}$ constant by adjusting the plasma current accordingly from $1.0 \mathrm{MA}-1.5$ MA. As the non-dimensional quantities $\rho^{*}$ and $\beta_{t h}$ scale as

$$
\begin{aligned}
\rho^{*} & \sim \frac{\sqrt{T}}{B} \\
\beta_{t h} & \sim \frac{n T}{B^{2}}
\end{aligned}
$$

the ratio of $\frac{T}{B^{2}}$ and the densities $n$ need to be kept constant for ions and electrons in an ideal scan by adjusting the heating power [22].

An overview of plasma parameters in the three-point scan is given in table 2. 
The collisionality was calculated at the pedestal top via [21]

$$
v_{e}^{*}=6.921 \cdot 10^{-18} \cdot q_{95} \cdot R_{\text {Surf }} \cdot n_{e, p e d} \frac{\left(1+30 \cdot \frac{n_{C, p e d}}{n_{e, p e d}}\right) \cdot\left(31.3-\ln \frac{n_{e, p e d}{ }^{0.5}}{T_{e, p e d}}\right)}{T_{e} \cdot\left(\frac{a}{R_{\text {Surf }}}\right)^{1.5}},
$$

where $R_{\text {Surf }}$ is the major radius of the center of the outermost closed flux surface, $a$ the minor radius and $Z_{\text {eff,ped }}$ the effective charge at the pedestal top. As $\frac{T}{B^{2}}$ and $\mathrm{n}$ are kept constant in the scan, $v_{e}^{*} \sim \frac{1}{B^{4}}$. A combination of $\mathrm{NBI}$ and $\mathrm{ECH}$ power steps was implemented within each discharge to investigate a wide variety of pedestal conditions. Typically, two to three time segments with stationary ELM cycle conditions (defined by small variations in $f_{E L M}$ and $p_{e, p e d}$ before ELM crash) were obtained per discharge, each lasting at least $0.5 \mathrm{~s}$. Strong heating power in the first segment ensured a stable L-H transition and high-quality data with beam-dependent diagnostics (most important: Charge Exchange Recombination (CER), Motional Stark Effect (MSE), Beam Emission Spectroscopy (BES)). The last segment in the discharges, when the input power was typically dropped to $\mathrm{P}_{\mathrm{inj}}=2.5$ - $3 \mathrm{MW}$, allowed investigation of conditions close to the LH-power threshold. The evolution of a medium-collisionality discharge is shown in figure 2 with steps in $\mathrm{P}_{\mathrm{NB}}$ at $\mathrm{t}=2.0$ $\mathrm{s}$ and $\mathrm{P}_{\mathrm{NB}}$ and $\mathrm{P}_{\mathrm{EC}}$ at $\mathrm{t}=3.5 \mathrm{~s}$ (Fig. 2c). The change of the stored energy at $3.5 \mathrm{~s}$ (Fig. 2a) is a response to the heating power change. The density pump-out effect of the $\mathrm{ECH}$ can be seen at $\mathrm{t}=3.5 \mathrm{~s}$, when both line-averaged and pedestal electron density increase after the ECH is switched off (Fig. 2b). In this discharge, the outer strike point is moved inward at $2 \mathrm{~s}$, i.e. away from the cryopump baffle, to allow proper measurement by IR camera. Here, three quasi-stationary intervals with different pedestal conditions were obtained: $1.5-2.0$ s (only inner divertor IR data), 2.2-3.4 s and $4.3-5$ s. $p_{e, p e d}$ remains approximately constant at ECH turn-off, with the increase in density resulting from the loss of $\mathrm{ECH}$ pump out balancing the decrease in temperature resulting from cessation of ECH heating (Fig. 2d). On the other hand, the density increase and the temperature decrease at 3.5 s cause a rise in collisionality (Fig. 2d). While the frequency of the ELMs decreases, the absolute size only changes marginally. In this discharge the power in the inner divertor during ELMs is about $5 \mathrm{MW}$ higher than the power arriving in the outer divertor (Fig. 2 e,f). 
During the experiment, a relatively good match of non-dimensional parameters was obtained as exemplified by figure 3 , where kinetic profiles of electron and impurity ion temperatures ( $a$ and c) normalized to $B^{2}$ and electron pedestal densities (b) are compared in $\psi_{N}$-space $\left(\psi_{N}(x)=\right.$ $\left.\frac{\psi(x)-\psi(0)}{\psi(s e p)-\psi(0)}\right)$. One representative of each collisionality point (table 2 ) is selected. The electron density profiles (b) agree well. Both the normalized electron and ion temperature profiles show that the low collisionality case (blue, 2.15T) has slightly lower normalized temperature values than required for perfect non-dimensionality match. The discrepancy exemplifies a common operational issue, i.e. the conflict of competing effects between increasing the power to improve the matching and undesirable high ELM frequencies due to the rise in SOL power. High ELM frequencies come with lower ELM energies and reduce the ELM size determination accuracy, as inter-ELM and ELM phase are more difficult to distinguish, among others. Additionally, the natural ELM frequency expected for ITER is below $10 \mathrm{~Hz}$ [5]. In high collisionality plasmas, there is a competition between lowering the heating power correctly with field and current and having enough beam power to maintain the beam dependent diagnostics, such as CER. As consequence of these competing goals, the pedestal beta in general reached higher values in the medium and high collisionality phase than desired for an ideal scan (table 2).

\section{Diagnostics}

The ELM heat flux was measured by infrared thermography, with an IR camera located at the top of the vessel and covering the lower divertor at a toroidal angle of 60 degrees [23]. For ELM measurements, a high time resolution is required to resolve the fast, transient changes in the divertor heat flux (in DIII-D, typical ELM rise time is $\sim 0.3 \mathrm{~ms}$.). The camera data was acquired in "line-mode", allowing frame rates of $12 \mathrm{kHz}$ with an integration time of $0.06 \mathrm{~ms}$, appropriate to resolve the observed ELM dynamics. The thermography analysis is based on the heat diffusion code THEODOR [24]. The code assumes the presence of surface layers, in poor contact with the bulk of tiles. The surface layer characteristics are required as a manual input into the code. Since different surface-layer assumptions can result in large corrections to the computed heat flux, 
determining the appropriate surface layer properties is a crucial step in the IR analysis. A detailed discussion on the procedure applied in this work to determine the DIII-D surface layers is included in appendix A. Notice that, while the divertor IR camera allowed accurate resolution of the ELM dynamics, the limited toroidal coverage prevented investigation of toroidal asymmetries of the ELM heat flux deposition and accounting for main chamber heat deposition.

As an example, the results of the thermography data analysis are shown for a low collisionality plasma (169426) in figure 4, where the evolution of the peak heat flux $q_{\text {peak }}$ is shown for the five largest ELMs in the selected time interval $t=4.4-5.0$ s. Here, data for inner (a) and outer divertor (b) are shown and the time coordinate represents the time since the ELM onset. Figures $4 \mathrm{c}$ and $\mathrm{d}$, show the radial profiles of heat flux at the time of peak. $q_{\text {peak }}$ in this example is higher on the inner divertor than on the outer $\left(25 \mathrm{MW} / \mathrm{m}^{2}\right.$ vs $\left.18 \mathrm{MW} / \mathrm{m}^{2}\right)$, whereas the decay is slower on the outer divertor. Thus, the resulting target ELM energy densities are comparable (e). Note that due to uncertainties in the equilibrium construction during the ELM part of the heat flux from the outer divertor is mapped in the private flux region [25]. As the ELM energy density lacks a normalization to the integration time, it is important to define a standard for determining time integration extremes. In accordance with [1] the time limits are determined by a drop to $\frac{1}{e^{2}}$ of the peak power value relative to the background heat flux and yield divertor ELM durations between $0.9 \mathrm{~ms}$ and $1.6 \mathrm{~ms}$ on DIII-D (dashed blue lines in (a) and (b)). As visible in figure 4 (e)(f), similar values of target and parallel ELM energy densities are found for the selected ELMs. In general, the standard deviation for the peak parallel ELM energy densities is below 35 \% for all timeintervals used in the analysis.

Fast bolometer measurements on DIII-D allow estimates of the energy radiated during an ELM. The newly rebuilt 12 channel SXR-45 detector [26] was found to provide the best signal-to-noise ratio for ELM data. It operates with AXUV (Si) photodiode detectors and polka-dot (neutral density) ND filters at a sample frequency of $20 \mathrm{kHz}$. The diagnostic is localized at a single toroidal location of 45 degrees. In order to infer the total ELM radiation power, axisymmetric behavior is assumed.

For a higher time resolution during ELMs, the Thomson laser system was set to fire in 'bunch'mode, i.e. instead of firing the lasers in equidistant time steps, the diagnostic was programmed 
to fire the lasers back to back in short time intervals followed by a comparatively longer pause. This configuration allows time resolution as low as $0.15 \mathrm{~ms}$. The Charge Exchange Recombination (CER) diagnostic data was acquired with $2.5 \mathrm{~ms}$ time exposure time $(400 \mathrm{~Hz})$. In each discharge, over a selected time interval of $0.1 \mathrm{~s}$, the exposure time was further reduced to $0.5 \mathrm{~ms}(2 \mathrm{kHz})$. For determining the ELM plasma energy losses $W_{E L M}$ (upstream plasma losses), magnetic reconstructions at time intervals as small as $0.5 \mathrm{~ms}$ are used, based on the fast diamagnetic Mirnov coils and Thomson profiles. The ELM energy is calculated as the maximum difference in a $3 \mathrm{~ms}$ interval around the drop in the fast energy signal.

The time evolution of four different ELMs on various diagnostics covering the SOL and the divertor is shown in figure 5. High collisionality discharges (red, green) are compared to midcollisionality plasma (black, blue). The magnetic perturbation indicating the energy loss of the plasmas (a) occurs at about the same time as the current increase in the tile current array in the divertor (b) as previously reported [27]. While the time resolution of the tile current array can reach $500 \mathrm{kHz}$, it was optimized to measure disruption energies, resulting in signal to noise ratios insufficient for precise identification of the ELM onset. The particle losses across the last closed flux surface into the $S O L$ are accompanied by an increase in $D_{\alpha}$ line radiation (c) and a sharp spike in divertor radiation (d). Finally, when the bulk of the particles hits the divertor, an increased heat flux is measured by IR thermography in the two divertor legs $(e, f)$.

\section{Plasma energy and heating overview}

In order to investigate the dependence of $\varepsilon_{\|}$on $v_{e}{ }^{*}$ and $p_{e, p e d}$, quasi-stationary time intervals were selected, characterized by different magnetic configuration and heating schemes. For each time window, kinetic equilibria were reconstructed with the EFIT [28] code, using experimental profiles selected in the 80-99 \% inter ELM phase. Pedestal profiles are fitted using the standard hyperbolic tangent functions [29]. Since for small ELMs at frequencies $\mathrm{f}_{\mathrm{ELM}}>60 \mathrm{~Hz}$ the accuracy of the ELM energy determination is greatly reduced, only time windows where $\mathrm{f}_{\mathrm{ELM}}<60 \mathrm{~Hz}$ were 
retained. As mentioned before, compound ELMs are not included in the analysis presented in this work.

Table 3 compares the average valued of a selection of plasma parameters for the time intervals of interests, in particular the ELM frequency, the plasma energy loss $\mathrm{W}_{\mathrm{ELM}}$ and the relative ELM size to the total plasma energy. $\mathrm{W}_{\mathrm{ELM}}$ is obtained as an average over all type-I ELMs in the respective time window. The main information conveyed in table 3 is that the increase of relative ELM sizes above $8 \%$ correlates with decreases in heating power, as shown by rows highlighted in red.

Out of the new experiment, twelve discharges with a total of 27 ELM-data time windows form the base of the analysis, each window with different plasma edge conditions and ELM frequencies of up to $60 \mathrm{~Hz}$. The energy range of 23-102 kJ is typical for type-I DIII-D ELMs. An overview of all obtained type-I ELMs during this experiment (including the ones not considered for the ELM scalings due to $\mathrm{f}_{\mathrm{ELM}}>60 \mathrm{~Hz}$ ) in frequency - size space is shown in figure 6 . The figure shows each ELM's dependence on the SOL power PsoL, which, in this work, represents the difference between total injected heating power (including ohmic heating) and measured radiative losses.

The characteristic behavior of type I ELMs is the reciprocal relationship of ELM frequency $f_{E L M}$ and size $W_{E L M}$ - for given $P_{S O L}[14,30]$

$$
f_{E L M} \cdot W_{E L M}=c \cdot P_{S O L}
$$

with the constant $c=0.2-0.4$. The black and blue dashed lines in figure 6 represent lower and upper limits to the ELM loss power space for the experiment of this paper. The dashed blue line assumes $8 \mathrm{MW}$ of SOL power (for the high heating phases) and an ELM loss power of $40 \%$ (c=0.4), whereas the black dashed line assumes $20 \%$ ELM loss power of $3 \mathrm{MW}$ SOL power $(\mathrm{c}=0.2)$. The distribution of data points confirms the characteristic behavior of type-I ELMs for the ELMs selected for the analysis. In particular, nearly no ELMs with high frequency and high power are observed.

For each ELM, an energy balance can be computed by comparing the plasma energy loss $W_{E L M}$ with the energy arriving in the divertor $E_{\text {div }}$ and radiation energy measurements. An example is shown in figure 7 , where $E_{\text {div }}$ is plotted as a function of the $W_{E L M}$. Each data point in 
the figure represents the average type-I ELM energy in a time window with stationary plasma conditions. Here $E_{\text {div }}$ is the sum of energy arriving during the ELM in the inner and outer divertor. While for ELMs with $\mathrm{W}_{\mathrm{ELM}}<30 \mathrm{~kJ}$ most of the energy is transported to the divertor, the maximum of $E_{\text {div }}$ for ELMs with $\mathrm{W}_{\mathrm{ELM}}>60 \mathrm{~kJ}$ remains around $60 \mathrm{~kJ}$, indicating divertor energy saturation at levels of $40-60 \%$ of $W_{E L M}$ for large ELMs. This result is in qualitative agreement with previous measurements of ELM energies on other tokamaks [16,17]. Radiative energies were measured to be between $26 \mathrm{~kJ}$ and $50 \mathrm{~kJ}$, so that the sum of radiation energy and divertor energy approximately equals the plasma energy loss, proving consistency of the ELM energy balance.

Finally, in order to extend the dataset to include variations of plasma shape from the modified ISS (e.g. shapes with lower triangularity), data from eight NBI heated H-mode plasmas from previous experiments [31,32] was included in the analysis. All discharges have uncontrolled typeI ELMs and IR data on inner and outer divertor. They cover the following ranges: $\mathrm{P}_{\mathrm{NB}} 1.5-6 \mathrm{MW}$, $\beta_{N} 1.2-2.5, \delta=0.3-0.6, \mathrm{~B}_{\mathrm{T}}=1.7-2.1 \mathrm{~T}, \mathrm{I}_{\mathrm{p}}=1.1-1.6 \mathrm{MA}$, and $\mathrm{q}_{95}=3.1-4.4 . v_{e}{ }^{*}$ ranges from $0.3-$ 1.2 .

\section{Test of Eich Model on DIII-D}

\section{Comparison of model and experimental data}

The comparison of the experimental DIII-D data and the Eich model prediction is shown in figure 8. As discussed previously, the model predicted values are obtained from experimental profiles in kinetic equilibrium reconstructions. While the standard EFIT solves the Grad-Shafranov Equation solely based on measurements from Motional Stark Effect polarimetry (MSE) and magnetics, the kinetic EFIT is obtained as a self-consistent equilibrium solution additionally constrained by profile measurements (including the fast ion population obtained from ONETWO [33] or TRANSP $[34,35]$ ) and their entailed currents (primarily the bootstrap current $j_{b s}$ ). Using kinetic profiles instead of standard profiles improves accuracy and provides interesting quantities for regression analysis (bootstrap current, pedestal ion pressure,...). The experimental values in figure 8 are computed by averaging the IR heat flux measurements over the five largest ELMs in the respective time-interval. Within a significant scatter, the DIII-D dataset, as a whole, appears 
to be consistent with the model. The peak parallel ELM energy density is slightly higher on the inner divertor, which in most cases is due to a higher target heat flux on the inner divertor. The average ratio of peak heat flux on the inner to outer divertor is $1.1: 1$ during the experiment described in this paper. The experimental data range is found to lie between half and two times the model prediction. The lines in the figure are 1 and 3 times the model prediction encompassing the range of data seen on AUG and JET. Since plasma shape and the field ratio $\frac{B_{T}}{B_{p}}$ were kept approximately constant as part of the non-dimensional scan, the Eich model prediction implies a linear relation between $p_{e, p e d}$ and $\varepsilon_{\|}$. Such dependence was not observed experimentally in the dataset as illustrated in figure 9, which shows the ELM energy density on the inner divertor in dependence of electron pedestal pressure and collisionality. In the region between $6-7 \mathrm{kPa}$ with $v_{e} \approx 0.2$, there are high $\varepsilon_{\|}$values of up to $0.6 \frac{\mathrm{MJ}}{\mathrm{m}^{2}}$ next to low values in the $0.2 \frac{\mathrm{MJ}}{\mathrm{m}^{2}}$ range. For the discharges shown in figure 3 the low $\mathrm{B}_{\mathrm{T}}$ plasma (high collisionality, low pressure) has the highest $\varepsilon_{\|}$. Large ELM energy densities in the $0.4 \frac{\mathrm{MJ}}{\mathrm{m}^{2}}$ range are measured in the high collisionality region $\left(v_{e}{ }^{*} \geq 0.8\right)$ over a pressure range from 3-6 kPa (in these time windows the high collisionality resulted from a significant reduction of both NBI and ECH power).

Although a linear dependence of the ELM energy density on the pedestal pressure is not seen, the spread in the dataset remains consistent with the threefold spread observed in the comparison between the Eich model and the multi-machine database [1]. Understanding this spread in the multi-machine comparison to the Eich model is an important open question, since it might be associated with one or more hidden variables not included in the model. The dependence of ELM energy density on ELM size $W_{E L M}$, convective and conductive ELM nature, and proximity to the $\mathrm{L}-\mathrm{H}$ threshold will be investigated in the remainder of this section as possible causes of the data spread around the model prediction.

\section{ELM size}

The correlation between peak parallel ELM energy density on the inner divertor and plasma loss energy during ELMs based on fast stored energy changes $W_{E L M}$ for each of the inspected time 
intervals is shown in figure 10. There is a significant scatter in the data and the minimal observed ELM energy density increases with $W_{E L M}$. Notably, relatively high $\varepsilon_{\|}$values can be reached at small ELM sizes: A $40 \mathrm{~kJ}$ ELM can generate a peak in parallel ELM energy density of up to $\varepsilon_{\|} \sim 0.45$ $\frac{M J}{m^{2}}$, which is the lower limit of the $\varepsilon_{\|}$range for ELMs in the $100 \mathrm{~kJ}$ range. In the experiment described in this paper, the ELM energy density is linearly proportional to the total energy deposited in the divertor. For ELMs with small $W_{E L M}$ with large $\varepsilon_{\|}$(as in the $40 \mathrm{~kJ}-0.45 \frac{M J}{\mathrm{~m}^{2}}$ case) a large fraction of the ELM energy arrives in the divertor, implying that the radiative fraction is small. An example of different $W_{E L M}$ but same $\varepsilon_{\|}$is shown in figure 5: The mid-collisionality discharge (red) has smililar peak heat flux profiles as the high collisionality discharge (e,f). But the ELM size (not plotted here) differs by $30 \mathrm{~kJ}$, due to a much higher ELM radiation energy (d).

\section{Collisionality dependence and conductive/convective character}

By comparing profiles of temperature and density before and after the ELM, $W_{E L M}$ can be split into a conductive and a convective part. Previous studies in DIII-D density scans [15] found that, while the energy fraction of an ELM transported convectively is approximately constant, the conductive energy loss associated with the pedestal temperature drop increases reciprocally with collisionality or proximity to the Greenwald density limit. Following these results, the largest values of $W_{E L M}$ are expected at low collisionality as the pedestal temperature drop across an ELM reaches its largest magnitude.

The convective and conductive fractions of ELM transport were estimated using the following procedure (similar to the procedure used in [15]): Pedestal density and temperature in the preELM phase were obtained from Thomson measurements using mappings from kinetic EFITs (corresponding to the $80-99 \%$ inter-ELM phase), while the pedestal values for the post ELM phase were calculated based on standard EFITs as described below. This is necessary due to large uncertainties in post ELM kinetic equilibrium reconstructions (0-20\% inter-ELM phase): the MSE diagnostic incorrectly attributes ELM-driven SOL current to in-plasma currents [25] and the data scatter in the Thomson measurements is high due to separatrix movement and convective transport. In detail, linear fits in time were applied to Thomson temperature and density 
measurements near the pedestal top of the first and last $10-20 \%$ in the conditionally averaged inter ELM phase. The goal of these fits was to project the time-varying measurements of temperature and density to values at the onset of the ELM and at the start of recovery from the ELM. While the ELM cycle is a complex non-linear process, linear fits in time as originally applied in [15] agree reasonably well with the Thomson measurements. The percent change of pre- to post-ELM value was then subtracted from the pedestal density and temperature values of the pre-ELM kinetic profiles. The conductive and convective energies are estimated as follows

$$
\begin{aligned}
& W_{\text {cond }}=\frac{3}{2} \cdot V_{E L M} \cdot n_{e, a v} \cdot\left(T_{e, p r e}-T_{e, p o s t}\right), \\
& W_{\text {conv }}=3 \cdot V_{E L M} \cdot T_{e, a v} \cdot\left(n_{e, p r e}-n_{e, p o s t}\right),
\end{aligned}
$$

where $V_{E L M}$ is the plasma volume affected by the ELMs, the $T_{e}$ and $n_{e}$ refer to the values of the electron profiles taken at the- pedestal top before (pre) and after (post) an ELM. $T_{e, a v}$ and $n_{e, a v}$ refer to average values, i.e. $\left(T_{e, p r e}+T_{e, p o s t}\right) / 2$. As the ion parallel transport time is much slower than for electrons, the ion conductive losses can be neglected, and the ion convective losses are assumed to balance electron convective losses, maintaining ambipolar transport (hence the factor 3 in equation 10) [15]. $V_{E L M}$ is calculated by comparing Thomson temperature and density profiles before and after the ELM and finding the maximum penetration depth of the ELM in $\psi_{N}$-space. From the $\psi_{N}-V$ grid in the pre-ELM kinetic EFIT the affected volume $V_{E L M}$ is obtained. In most cases the profile comparison indicates a maximum penetration depth between $\psi_{N}=0.5$ and $\psi_{N}=0.6$, corresponding to an affected volume of approximately $10 \mathrm{~m}^{3}$. In some cases, notably for input powers close to the L-H power threshold, a penetration up to a minimal $\psi_{N}=0.3$ is found. The results are shown in figure 11: there is strong scatter, but the conductive energy loss (red) tends to increase at lower collisionality, confirming the results of previous ELM studies [15]; however, the convective loss (blue) doesn't show a clear correlation with collisionality (a). It should be noted though, that when comparing the ELM energy obtained by adding the convective and conductive energy fractions to the ELM energy loss measured by magnetics, $W_{E L M}$, the ELM energy inferred from the kinetic profiles tends to underestimate the measured ELM energy in the higher collisionality range. This could be associated with the pure 
plasma assumption: The high collisionality cases are in general closer to the LH-threshold where, as explained in the following section, the ELM induced density reductions are stronger for impurities than for electrons. As most plasmas in the experiment were operated at power input close to the LH power threshold, biasing the dataset towards large ELM sizes at high density (and high collisionality), the expected inverse proportionality between collisionality and fast MHD energy losses was not seen. The ELM size in the previous DIII-D study stayed below a relative level $\mathrm{W}_{\mathrm{ELM}} / \mathrm{W}_{\text {ped }}$ of $20 \%[15]$, whereas here values up to $35 \%$ are included. For this experiment the ratio of measured ELM energy loss $W_{E L M}$ and pedestal energy as defined in (4) does not depend on collisionality (Fig 11b).

Connecting back to Eich's model, the model underestimates the $\varepsilon_{\|}$for ELMs with large convective transport near the LH threshold, as will be explained in the following segment.

\section{LH threshold}

As shown in the ELM size overview of table 3, the largest relative ELM losses (red background) are seen with relatively small injected power at low ELM frequencies. The dataset indicates, that a change in ELM frequency alone does not necessarily imply a change in relative ELM size and that the heating power plays a strong role in determining relative ELM size. For instance, a doubling of the ELM frequency at constant power on 169425 (from $22 \mathrm{~Hz}$ to $45 \mathrm{~Hz}$, caused by a density change) only leads to a marginal change in ELM size. In discharge 169433, where the relative ELM size $\left(W_{E L M} / W_{M H D}\right)$ almost doubles following the $50 \%$ reduction of $P_{N B}$ from $\mathrm{t}=1.8$ $\mathrm{s}$ to $\mathrm{t}=3.0 \mathrm{~s}, f_{E L M}$ decreases over-proportionally from $25 \mathrm{~Hz}$ to $7 \mathrm{~Hz}$, further indicating a stronger role of the input power than $f_{E L M}$ as driver behind ELM size changes. Analysis of the dataset here suggests that proximity of the heating power to the $\mathrm{L}-\mathrm{H}$ threshold is a strong determinant of the relative ELM size. This analysis is illustrated in figure 12 which shows that the largest ELM losses $W_{E L M}$ relative to the total plasma energy of up to $14 \%$ occur when the heating power gets closer to the L-H threshold, defined by [36]

$$
P_{L H}=0.049 \bar{n}^{0.72} B_{T}^{0.8} S^{0.94}
$$


Here $S$ stands for the plasma surface in $\mathrm{m}^{2}, \bar{n}$ for the line-averaged electron density in $10^{20} \mathrm{~m}^{-3}$ and $B_{T}$ is the central magnetic field strength in T. The heating power $P_{\text {heat }}$ is the sum of ohmic and external heating by ECH and NBI. Since the plasma is in a stationary state during the inspected time intervals, there were no changes in plasma energy other than ELM losses, so $\frac{d W}{d t}$ was ignored in the $P_{\text {heat }}$ sum. As soon as $P_{\text {heat }}$ reaches 2.5 times the LH-threshold value, the relative plasma losses during ELMs stay below $10 \%$.

Not only ELM energies but also ELM energy densities increase closer to the L-H threshold, which can be seen with the aid of figure 9 . In this dataset, equilibria close to the L-H threshold are found at higher collisionality due to the low heating power and have large ELM energy densities (the figure also shows high ELM energy densities obtained at low collisionality). The amount of scatter in the Eich model correlates with the relative heating power. This scatter is shown in fig 13, which is a plot of the ratio of experimental ELM energy density to the Eich model prediction on the inner divertor versus the relative heating power. The inner divertor is chosen, as it generally had higher ELM energy densities during this experiment and more time windows can be included. Low densities ensure complete attachment and contrary to the outer divertor the full width is visible on the fast IR camera. Notice that previous standard H-mode experiments (see section 6) are included in this figure and confirm the trend found in the more recent experiments. The $\varepsilon_{\|}$range relative to the Eich Model prediction decreases from 0.5-2.5 for discharges marginally above the threshold to 0.8-1.2 for strongly heated plasmas. To conclude, the Eich model performs generally better in well heated plasmas. The physics behind the ELM size increase close to the LH threshold will be inspected in the following section. 


\section{ELM characteristics close to LH power threshold}

The proximity to the $\mathrm{LH}$ threshold power is found to have a strong influence on ELM behavior. A typical case demonstrating changes in the ELM dynamics when the input power approaches the $\mathrm{LH}$-threshold is shown in figure 14. Following a drop of $P_{N B}$ from 4.0 to $1.4 \mathrm{MW}$, the total heating power of the discharge including ohmic contributions amounts to $2 \mathrm{MW}$, which is approximately the LH-threshold power in this time window (a). The ELM frequency initially decreases from 23 to $16 \mathrm{~Hz}$ (for 200ms, yellow window), and subsequently drops to $f_{E L M} \sim 3 \mathrm{~Hz}$. While density profiles of electrons (e) and the Carbon impurities (f) are not affected in the intermediate phase, they start to increase in the low frequency phase. The confinement improvement is expressed by the $\mathrm{H}_{98}$ factor increase from $\mathrm{H}_{98} \sim 1.5$ to 1.7. These generally high confinement values could be achieved due to running the experiment closely after a boronization on DIII-D. Similarly, the beta values decrease marginally during the intermediate phase and then rise in the low frequency phase. The pedestal width increases during the beta rise phase. By comparing the total plasma energy $W_{M H D}(\mathrm{~b})$, the pedestal pressure (e), and the peak heat flux $q_{\text {peak }}$ on the inner divertor (c), it becomes evident that the relative ELM size is largest in the third, low frequency phase (notice that both plasma energy and pedestal pressure are lower than in the first segment, but $q_{p e a k}$ and $W_{E L M}$ are similar). It is interesting to investigate the characteristics of these ELMs in comparison to ELMs in time intervals characterized by larger input power and larger ELM frequency. A comparison of electron and ion profiles before and after the ELM (figure 15) shows that the largest difference comes with the electron and carbon impurity density profiles. First, note that the post ELM impurity density profiles look similar. In contrast, the pre-ELM profile is about $50 \%$ higher at the edge when closer to the LH threshold. Similarly, the edge electron density decrease is stronger. These combined effects result in a large loss of bootstrap current, obtained when comparing pre- and post-ELM kinetic EFITs, as its $j_{b s}$ is largely determined by the pedestal density gradient in these discharges [21].

Linear stability analysis was performed for pre-ELM profiles taken before and after the NBI power reduction. The results are represented with the peeling-ballooning stability map (discharge 
169509 , figure 16), in which the $x$-axis represents $\alpha$, the normalized pressure gradient, defined as [37]

$$
\alpha(r)=-\frac{2 \mu_{0} R}{B^{2}} \cdot q^{2} \frac{d p}{d r}
$$

The plotted value is the maximum of $\alpha(r)$ in the plasma edge. The $y$-axis denotes the relative edge current density $j_{\text {norm }}$, defined by the ratio of the sum of the maximum current density in the edge region $j_{\text {edge,max }}$ and the separatrix current density $j_{\text {sep }}$ to the normalized current density across the plasma $j_{a v}$

$$
j_{\text {norm }}=\frac{j_{\text {edge, } \max }+j_{\text {sep }}}{2 j_{\text {av }}}
$$

The computation finds that, with reduced input power the changes in pedestal stability are minimal: the experimental points sit in similar locations of the stability map, the stability boundary itself changes marginally, and the linear growth rate spectra for both equilibria are similar, with $n=16$ being the most unstable mode number.

In the combined framework of the peeling ballooning model [38] and ELM loss power as a constant share of SOL power (described in equation 8), the ELM behavior in the intermediate period (yellow) can be understood as a consequence of the NBI power reduction: The heating power loss leads to a slower pedestal buildup towards the same pedestal critical conditions, resulting in a lower ELM frequency. Indeed, as the ELM loss power is reduced due to the smaller SOL power, the ELM energy loss remains approximately constant. In contrast, the observation of large ELM sizes despite lower heating and smaller pedestal energy in the third section of the discharge is more difficult to interpret. In this case, where the power input nears the $\mathrm{P}_{L H}$ threshold, the peeling ballooning model offers no insight as to why the ELM frequency rapidly drops from $16 \mathrm{~Hz}$ to $3 \mathrm{~Hz}$. 


\section{Relation between ELM sizes and results of linear stability analysis}

ELM energy densities are found to scale inversely with the calculated most unstable toroidal linear mode number on this DIII-D dataset. The peeling-ballooning model for the ELM onset has proven to be successful in analyzing the linear stability of ELMy H-mode plasmas, as well as ELM controlled scenarios as QH mode and RMP [39-41]. Based on the extensive validation, the model is presently used to extrapolate pedestal stability in future machines like ITER [42]. The model addresses the linear stability of the pedestal, providing information on the mode structure during the initial exponential growth, which precedes the non-linear phase, associated with the pedestal collapse [43]. In principle the amplitude of the ELM losses should be addressed by non-linear MHD simulations [44], which require extremely demanding computation resources. Consequently, there have been efforts to link linear simulations to non-linear quantities $[45,46]$. Here, the question is raised whether the results of the linear ELITE code can provide insight about a non-linear quantity, such as the ELM size. For instance, a common conjecture, based on extensive set of ELITE simulations, associates low-n peeling-ballooning modes with larger ELM sizes, due to the broad radial eigenfunction [42].

In this work, stability analysis with the ELITE code was performed on all 27 kinetic equilibria of the dataset, to determine whether the position in the PB stability map, the toroidal mode number of the most stable modes, or growth rates have an influence on the experimentally observed ELM sizes. The latest version of the code was used including a bi-linear fit model for the calculation of the effective diamagnetic stabilization rate [47] to improve the accuracy of the results.

To extend the range of the most unstable mode number $n_{\max }$ up to high values typical of the ballooning regime, the discharges from previous experiments mentioned in section 3 were included in the analysis. From this additional dataset, we selected four discharges representing a shot-by-shot density scan, which was obtained by means of gas puffing and variation of heating power (shape, field and current were held constant). For this dataset, a wide region in the typeI ELM region from low- $n$ to high-n PB modes is covered with similar plasma conditions $\left(W_{M H D}, \beta_{N}, p_{e, p e d}, \delta\right)$. Different from the experiment of this paper, the outer strike point is on the shelf (elevated platform in lower divertor, shown on the lower right in figure 1 with "modified 
ISS") and the plasma current is 1.3 MA. Figure 17 shows the ELITE results from three consecutive discharges from this density scan. The stability boundary (for an equilibrium on this boundary the mode growth rate $\gamma_{n}$ equals the effective stabilization rate $\left.\gamma_{\omega i, e f f}\right)$ in the standard PB map is drawn as a contour line for each of the discharges. The color of each stability boundary corresponds to $\varepsilon_{\|}$(from the inner or outer divertor, whichever is larger). The exact position of the equilibrium in $\mathrm{j}-\alpha$ space is denoted by the cross in the respective color. An error bar of $\pm 10 \%$ is applied to account for the uncertainties in pedestal pressure and edge current density. From the position of the cross relative to the respective stability boundary one can distinguish the three discharges as low-n (yellow), intermediate-n (green), and high-n (purple) peeling-ballooning unstable. As summarized in table 4 , the lowest $\varepsilon_{\|}=0.26 \mathrm{MJ} / \mathrm{m}^{2}$ is measured during the most ballooning-unstable ( $n=35$ ) plasma, and the highest $\varepsilon_{\|}=0.43 \mathrm{MJ} / \mathrm{m}^{2}$ is measured during the most peeling-unstable $(n=14)$ plasma. The relative growth rates of the most unstable mode $\frac{\gamma_{n}}{\gamma_{\omega i, e f f}}$ are similar for these equilibria.

There are three trends for the density scan experiment shown in this table:

- While the normalized growth rate determines how unstable a reconstructed equilibrium is, it does not correlate with ELM energy density. Typical type I ELMs are in the range of 0.8-1.5 around the onset of the instability.

- $\quad \varepsilon_{\|}$increases with lower $n_{\max }$. This could either be due to the deeper penetration of the lower $n$ modes into the plasma in the linear phase or due to the stronger role of the edge current. Analysis of numerous discharges did not show a correlation between edge current (relative and absolute size) and $\varepsilon_{\|}$, while the inverse scaling of $\varepsilon_{\|}$and $n_{\max }$ held for all inspected discharges as explained below.

- The prediction of Eich's model is less accurate at lower $n_{\max }$. The physics causing the change in linear mode number structure (i.e. change of collisionality and effects of $P_{L H}$ proximity) is not captured in the model. Further discharge analysis will show that there is no general dependence of the Eich scatter on mode numbers. 
Figure 18 shows peak ELM energy densities from the experiment of this paper (including additional discharges described in section 3) as a function of the most unstable linear mode number $n_{\text {max }}$. As the ELITE calculation of mode numbers is sensitive to changes in the profiles of the equilibrium reconstruction, an error bar was added based on all mode numbers with growth rates larger than $90 \%$ of the $n_{\max }$ growth rate. Aside from the current profile, the exact location of the separatrix and the resulting electron temperature in this region are the largest sources of uncertainty. Because the comparison of the five ELMs over which the heat flux is averaged shows good reproducibility (Fig 4), a $10 \%$ error bar is assumed for the IR measurement. The results indicate a correlation with decreasing trend (as can be seen in the $1.1 \cdot n_{\max }{ }^{-0.38}$ fit to the data), with the largest ELM loads being reached for low-n peeling-ballooning modes. This confirms the findings of the density scan analysis (table 4) and offers a better interpretation of the role of collisionality: for cases in which lower collisionality or a different edge density gradient lead to a lower $n_{\text {max }}$, lower $v_{e}^{*}$ will come with larger ELM energy densities and ELM sizes. But these large ELM energy densities can also be reached at high collisionality if $n_{\max }$ is lowered by another quantity, e.g. a large density gradient at the edge facilitating a strong bootstrap current. Regarding comparisons to other tokamaks, it is highly desirable to find a normalization quantity for $\varepsilon_{\|}$; at present a reasonable non-dimensional normalization for a $\varepsilon_{\|}=f(X) \cdot n_{\max }{ }^{-c}$ relation (with c a constant and $f(X)$ a function of plasma parameter) has not been found yet.

Identifying the exact physics mechanism behind the ELM energy density increase at lower mode numbers is beyond the scope of this work. As previously stated, a possible explanation is that lower-n-PB modes penetrate deeper into the plasma increasing the ELM size in the divertor. Radial eigenfunctions of the $n_{\max }=14$ and $n_{\max }=35$ equilibria are shown in figure 19 . One can see that in the $n_{\max }=14$ case, there is a considerable kink tail and the penetration reaches to about $\psi_{N}=0.6$, while the high- $n_{\max }$ case only penetrates to $\psi_{N}=0.8$.

The scatter in the experimental data relative to the Eich model prediction can now be interpreted as a consequence of only including the pedestal height of all quantities considered in linear stability analysis. The prediction accuracy of the Eich model will decrease as soon as additional actuators (as triangularity, beta, collisionality ...) cause a change in mode numbers without 
altering the $p_{e, p e d}$. These "hidden variables" are responsible for the scatter in the Eich model, with the L-H threshold proximity being important for the largest outliers in this study.

Experimental signatures of this trend (thermal profiles, rotation) could unfortunately not be produced, as the fast CER measurements of ELMs during the experiment were inconclusive due to an insufficient signal-to-noise ratio in the $0.25 \mathrm{~ms}$ integration period. From Thomson measurements a weak correlation between the most unstable mode number and the penetration depth of the electron temperature perturbation is seen; that is, deeper penetration of the temperature perturbation was typically associated with lower most unstable mode numbers. Some ELMs, having low $n_{\max }$ and short ELM penetration depth, deviate from this picture. However, these ELMs exhibit stronger decreases in electron temperatures in the ELM affected space. 


\section{Summary and Discussion}

A non-dimensional shot-by-shot collisionality scan has been carried out on the DIII-D tokamak to investigate the scaling of ELM heat loads with pedestal pressure and collisionality and test the Eich model for ELM energy densities. The dataset collected includes 27 time windows with well

diagnosed ELM activity spanning a range from $v_{e}^{*}=0.05-2.17$ and $p_{e, p e d}=3.2-7.8 \mathrm{kPa}$. The dataset has been extended to include other $\mathrm{H}$-mode discharges with collisionalities up to 1.2. In general, for large ELMs between $40 \%$ and $60 \%$ of the ELM energy is deposited onto divertor tiles, with peak heat flux only slightly higher at the inner divertor than the outer (ratio in/out: 1.1 : 1). Bolometry measurements during ELM crashes are consistent with a large fraction of ELM energy (up to $\sim 50 \%$ ) being dissipated through radiation. Hence, the ELM energy balance suggests that the direct heat deposition on the main chamber wall is small in these cases. The main results of the experiment are briefly summarized here:

- As a whole, the measured ELM energy densities appear consistent with the prediction of Eich model. However, a clear correlation between the model prediction and experiment is not found. The measured $\varepsilon_{\|}$covers the range between 0.5-2 times the predicted values.

- The non-dimensional collisionality scan did not reveal an increase of $\varepsilon_{\|}$with $v_{e}^{*}$. On the contrary, for high collisionalities near the LH power threshold, the Eich model yielded predictions of $\varepsilon_{\|}$values which significantly under-estimated the experimental values.

- The prediction of Eich's model are found to be more accurate when the heating power is much larger than the LH power threshold $(>2 \mathrm{X})$. In particular, for the same pedestal pressure, much larger ELM energies are observed for low-frequency ELMs found for $P_{\text {heat }} \sim P_{L H}$. This suggest that, to improve the predictive capabilities in this regime, a model should account not only for the pedestal properties at ELM crash, but also for the dynamic evolution of the pedestal towards the ELM crash.

For the dataset in consideration, an inverse correlation is found between the peak parallel ELM energy density and the most unstable toroidal mode number calculated by linear MHD stability 
analysis. Considering the scaling $W_{E L M} \sim \frac{1}{v}$ derived in [12] through an extensive multi-machine analysis, this result suggests that lower collisionality would be correlated with lower toroidal mode numbers. This is intuitively acceptable, given that low collisionality generally results in low $\mathrm{n}$ peeling modes, associated with higher bootstrap current. A clear correlation between collisionality and mode number did not emerge from the present dataset. It is interesting to investigate how the observations in the experiments can affect the projection to ITER, regarding the two models considered in this work: Eich's model (ELM energy density scales with $p_{e, p e d}$ ) and Loarte's model (Elm energy scales with $v_{e}^{*}$ ). The predictions of the two models for ITER have been carried out in section 2, showing a small discrepancy in the non-active phase of ITER operation $\left(I_{P}=7.5 \mathrm{MA}, B_{T}=2.65 \mathrm{~T}\right)$, and being significantly different during the active phase $\left(I_{P}=\right.$ $\left.15 \mathrm{MA}, B_{T}=5.3 \mathrm{~T}\right)$.

During the DIII-D experiments two observations were made which can raise concern for future ITER operation:

- First, for plasmas with $\mathrm{P}_{\text {heat }} \mathrm{P}_{L H}$ ELMs with $\mathrm{W}_{\mathrm{ELM}} / \mathrm{W}_{\mathrm{MHD}}$ up to $14 \%$ were robustly documented, which challenges the predictions of both Loarte and Eich's models. Since ITER will operate with $P_{\text {heat }}>=P_{L H}$, this suggests that the model predictions might not be accurate: We first consider the non-active phase of ITER operation: assuming a magnetic field of $2.65 \mathrm{~T}$, line-averaged density $\bar{n}=0.8 \cdot 10^{20} \frac{1}{\mathrm{~m}^{3}}$ and plasma cross section of 620 $\mathrm{m}^{2}$ the projected $\mathrm{L}-\mathrm{H}$ - threshold is $\mathrm{P}_{\mathrm{LH}}=38 \mathrm{MW}$. In the optimistic scenario, $\mathrm{P}_{\text {heat }}=$ $\mathrm{P}_{\mathrm{OH}}+\mathrm{P}_{\mathrm{ICRH}}+\mathrm{P}_{\mathrm{ECH}}+\mathrm{P}_{\mathrm{NB}}=73 \mathrm{MW}$ (assuming $\mathrm{P}_{\mathrm{NB}}=30 \mathrm{MW}$ ), results in $\mathrm{P}_{\text {heat }} \sim 1.9 \mathrm{P}_{\mathrm{LH}}$. Assuming a plasma energy of $100 \mathrm{MJ}$ for ITER's non-active phase, a 14 \% ELM would be 3 times above the currently assumed $4 \mathrm{MJ}$ for uncontrolled type-I ELMs in this phase [5].

- Second, it was shown that the ELM energy density inversely correlates with $n_{\max }$, the toroidal mode number with highest growth rate computed from linear stability. If the inverse scaling between $n_{\max }$ and $\varepsilon_{\|}$is a general result, i.e. confirmed by experiments on other devices, it would provide a way to extrapolate the ELM energy densities from linear stability calculations of ITER operation equilibria.

The experimental results described in this work challenge the present-day models of ELM heat 
load scaling and strongly motivate further experimental and modeling research. Future work in this area should focus on ELM activity close to the LH threshold, with particular emphasis on the role of pedestal micro-instabilities in determining the pedestal evolution towards the ELM crash. Furthermore, effort should be put in testing the relation between the linear mode number and ELM energy density, in other plasma conditions and other tokamaks. If confirmed and understood, the scaling could provide an effective tool to estimate the size of ELM events on present machines and ITER, without the recourse of challenging non-linear MHD simulations. 


\section{APPENDIX}

A. IR measurements

As deducing heat fluxes from IR measurements comes with large uncertainties, the process will be briefly described here. The IR camera photon count rate is translated into temperature information using the Planck law for a gray body. The THEODOR code [24] is used to calculate the heat fluxes causing the temperature changes.

An important aspect of any thermography analysis is the characteristic of the surface layers. In fact, dust deposits with poor heat conduction with the tiles can substantially affect the temperature dynamics, which, if not properly accounted for, can lead to overestimates and, in some cases, negative heat fluxes. In case of the latter, the code does not properly account for heat dispersion abilities provided by the large surface of the layers; in the former case the additional photons emitted by the surface layer atoms are wrongly attributed to the tiles. To account for the changed morphology of the tiles in the THEODOR code, one must estimate the ratio of their conductivity to thickness, known as the $\alpha$ coefficient. To find the best parameter an optimization was undertaken along three competitive goals:

1. The energy balance during the ELMs and the discharge as a whole must be physical, i.e. there cannot be more energy in the divertor than injected by ohmic and external heating

2. At the end of the discharge, the energy in the divertor must remain constant as the total arriving power is zero

3. The temporal shape of the ELMs should ideally resemble a large initial spike, followed by a valley during which the pedestal is restored

For the discharges inspected in this paper, $\alpha=75000$ was determined to be the optimal value for meeting the above goals. The main uncertainty emerges from averaging over the nonaxisymmetric ELM behavior at only one toroidal measurement location. 


\section{TABLE CAPTIONS}

\begin{tabular}{|l|c|c|c|c|c|}
\hline \multicolumn{2}{|c|}{$\varepsilon_{\text {tar }}\left[\frac{M J}{m^{2}}\right]$} & \multicolumn{2}{c|}{ Eich } & \multicolumn{2}{c|}{ Loarte } \\
\cline { 3 - 6 } & Lower limit & Upper limit & $3 \times$ Broadening & No \\
broadening \\
\hline \multirow{2}{*}{$\begin{array}{l}\text { Non-active phase } \\
\text { (W }\end{array}$} & In & 0.41 & 1.23 & 0.37 & 1.10 \\
\cline { 2 - 6 } & Out & 0.12 & 0.36 & 0.11 & 0.32 \\
\hline Active phase & In & 1.78 & 5.34 & 3.67 & 11.0 \\
\cline { 2 - 6 }$\left(\mathrm{W}_{\text {ped }}=100 \mathrm{MJ}\right)$ & out & 0.52 & 1.56 & 1.08 & 3.2 \\
\hline
\end{tabular}

Table 1 Comparison of Eich and Loarte model for ITER's ELM energy densities, the recommended material limit is $0.15 \mathrm{MJ} / \mathrm{m}^{2}$

\begin{tabular}{|c|c|c|c|}
\hline & $\begin{array}{l}\text { Low } \\
\text { collisionality }\end{array}$ & $\begin{array}{c}\text { Medium } \\
\text { collisionality }\end{array}$ & $\begin{array}{l}\text { High } \\
\text { collisonality }\end{array}$ \\
\hline$B_{T}[\mathrm{~T}]$ & 2.15 & 1.80 & 1.60 \\
\hline$I_{P}[\mathrm{MA}]$ & 1.50 & 1.26 & 1.12 \\
\hline$P_{N B}[\mathrm{MW}]$ & $1.6-5.0$ & $1.6-5.0$ & $1.6-3.0$ \\
\hline$P_{E C}[\mathrm{MW}]$ & $0-3.5$ & $1.6-2.3$ & $0-2.2$ \\
\hline $\boldsymbol{v}_{\boldsymbol{e}}^{*}$ & $0.05-0.75$ & $0.13-0.34$ & $0.45-2.17$ \\
\hline$f_{E L M}[\mathrm{~Hz}]$ & $7-47$ & $14-31$ & $8-43$ \\
\hline$p_{\text {ped }}[\mathrm{kPa}]$ & $4.2-6.5$ & $3.9-7.8$ & $3.1-4.8$ \\
\hline$\beta_{n, p e d}$ & $0.45-0.56$ & $0.47-0.91$ & $0.52-0.72$ \\
\hline
\end{tabular}

Table 2. Operational overview of three-point collisionality scan 


\begin{tabular}{|c|c|c|c|c|c|c|c|c|}
\hline $\begin{array}{c}\text { discharge } \\
\mathrm{B}_{\mathrm{T}}(\mathrm{T})\end{array}$ & $t(s)$ & $\pm \Delta t(s)$ & $\begin{array}{l}P_{E C H} \\
(\mathrm{MW})\end{array}$ & $\begin{array}{l}P_{N B I} \\
(\mathrm{MW})\end{array}$ & $\begin{array}{c}f_{E L M} \\
(\mathrm{~Hz})\end{array}$ & $\begin{array}{c}W_{M H D} \\
\text { (MJ) }\end{array}$ & $\begin{array}{c}W_{E L M} \\
(\mathrm{~kJ})\end{array}$ & $\frac{W_{E L M}}{W_{M H D}}(\%)$ \\
\hline \multirow{3}{*}{$\begin{array}{c}169425 \\
2.15\end{array}$} & 2.8 & 0.25 & 3.3 & 3.7 & 22 & 1.09 & 60 & 5.5 \\
\hline & 3.2 & 0.2 & 3.3 & 3.8 & 45 & 1.04 & 59 & 5.6 \\
\hline & 4.2 & 0.4 & 2.1 & 0.6 & 21 & 0.73 & 59 & 8.1 \\
\hline \multirow{2}{*}{$\begin{array}{c}169426 \\
2.15\end{array}$} & 4 & 0.35 & 3.3 & 1.9 & 35 & 0.81 & 61 & 7.5 \\
\hline & 4.7 & 0.3 & 3.3 & 1.9 & 33 & 0.81 & 73 & 8.9 \\
\hline \multicolumn{9}{|l|}{169427} \\
\hline 2.15 & 1.8 & 0.2 & 3.5 & 4.6 & 67 & 1.18 & 23 & 1.9 \\
\hline \multirow{3}{*}{$\begin{array}{c}169430 \\
1.6\end{array}$} & 1.8 & 0.25 & 1 & 2.8 & 20 & 0.67 & 43 & 6.5 \\
\hline & 2.9 & 0.55 & 1 & 1.5 & 17 & 0.71 & 62 & 8.7 \\
\hline & 4.3 & 0.55 & 0 & 2.4 & 11 & 0.81 & 83 & 10.2 \\
\hline \multirow{3}{*}{$\begin{array}{c}169431 \\
1.8\end{array}$} & 2.4 & 0.2 & 2.2 & 4.3 & 14 & 1.14 & 95 & 8.4 \\
\hline & 3.2 & 0.24 & 2.2 & 4.3 & 21 & 1.00 & 81 & 8.1 \\
\hline & 4.3 & 0.55 & 2.2 & 1.6 & 27 & 0.70 & 68 & 9.7 \\
\hline 169432 & 2.7 & 0 & 0 & 1.3 & 10 & 0.54 & 68 & 12.5 \\
\hline 1.6 & 4.3 & 0.4 & 0.6 & 2.4 & 7 & 0.76 & 87 & 11.4 \\
\hline \multirow{3}{*}{$\begin{array}{c}169433 \\
1.6\end{array}$} & 1.8 & 0.25 & 1.1 & 2.8 & 25 & 0.66 & 43 & 6.6 \\
\hline & 3 & 0.75 & 1.1 & 1.3 & 7 & 0.66 & 70 & 10.6 \\
\hline & 4.8 & 0.55 & 1 & 2.4 & 15 & 0.46 & 62 & 13.5 \\
\hline \multirow{3}{*}{$\begin{array}{c}169434 \\
1.8\end{array}$} & 2.3 & 0.2 & 1.7 & 5 & 14 & 1.17 & 99 & 8.5 \\
\hline & 3 & 0.48 & 1.7 & 4.8 & 18 & 1.07 & 102 & 9.6 \\
\hline & 4.2 & 0.35 & 1.3 & 1.3 & 19 & 0.62 & 56 & 9.0 \\
\hline \multirow{3}{*}{$\begin{array}{c}169508 \\
2.15\end{array}$} & 2.8 & 0.65 & 3.3 & 4.5 & 17 & 1.19 & 28 & 2.4 \\
\hline & 4 & 0.2 & 3.3 & 1.7 & 22 & 0.92 & 66 & 7.2 \\
\hline & 4.8 & 0.25 & 3.3 & 1.7 & 38 & 0.76 & 47 & 6.1 \\
\hline \multirow{2}{*}{$\begin{array}{c}169509 \\
2.15\end{array}$} & 3 & 0.4 & 0 & 4 & 14 & 1.22 & 85 & 6.9 \\
\hline & 4.2 & 0.35 & 0 & 1.4 & 4 & 0.99 & 89 & 9.0 \\
\hline 169510 & 2.2 & 0.25 & 3.2 & 4.6 & 20 & 1.12 & 35 & 3.1 \\
\hline
\end{tabular}




\begin{tabular}{|l|l|l|l|l|l|l|l|l|}
\hline \multirow{2}{*}{2.15} & 3.1 & 0.3 & 0 & 4.5 & 10 & 1.27 & 89 & 7.0 \\
\cline { 2 - 9 } & 4.2 & 0.35 & 0 & 1.7 & 16 & 0.95 & 59 & 6.2 \\
\hline
\end{tabular}

Table 3 Selected discharge time windows with heating powers in MW, ELM frequencies and sizes. Rows in red mark time windows with relative ELM sizes above $9 \%$.

\begin{tabular}{|c|c|c|c|}
\hline $\begin{array}{c}\text { Most unstable } \mathrm{n} \\
\text { calculated }\end{array}$ & $\begin{array}{c}\text { ELM energy density } \\
\text { experimental }\end{array}$ & $\begin{array}{c}\frac{\gamma_{n}}{\gamma_{\omega i, \text { eff }}} \\
\text { calculated }\end{array}$ & $\begin{array}{c}\frac{\varepsilon_{\text {exp }}}{\varepsilon_{\text {Eich }}} \\
\text { experimental }\end{array}$ \\
\hline 14 & 0.43 & 1.5 & 1.7 \\
\hline 15 & 0.39 & 1.9 & 1.6 \\
\hline 17 & 0.38 & 1.2 & 1.6 \\
\hline 24 & 0.30 & 1.4 & 1.3 \\
\hline 35 & 0.26 & 1.4 & 1.1 \\
\hline
\end{tabular}

Table 4 Linear stability analysis results of density scan experiment 


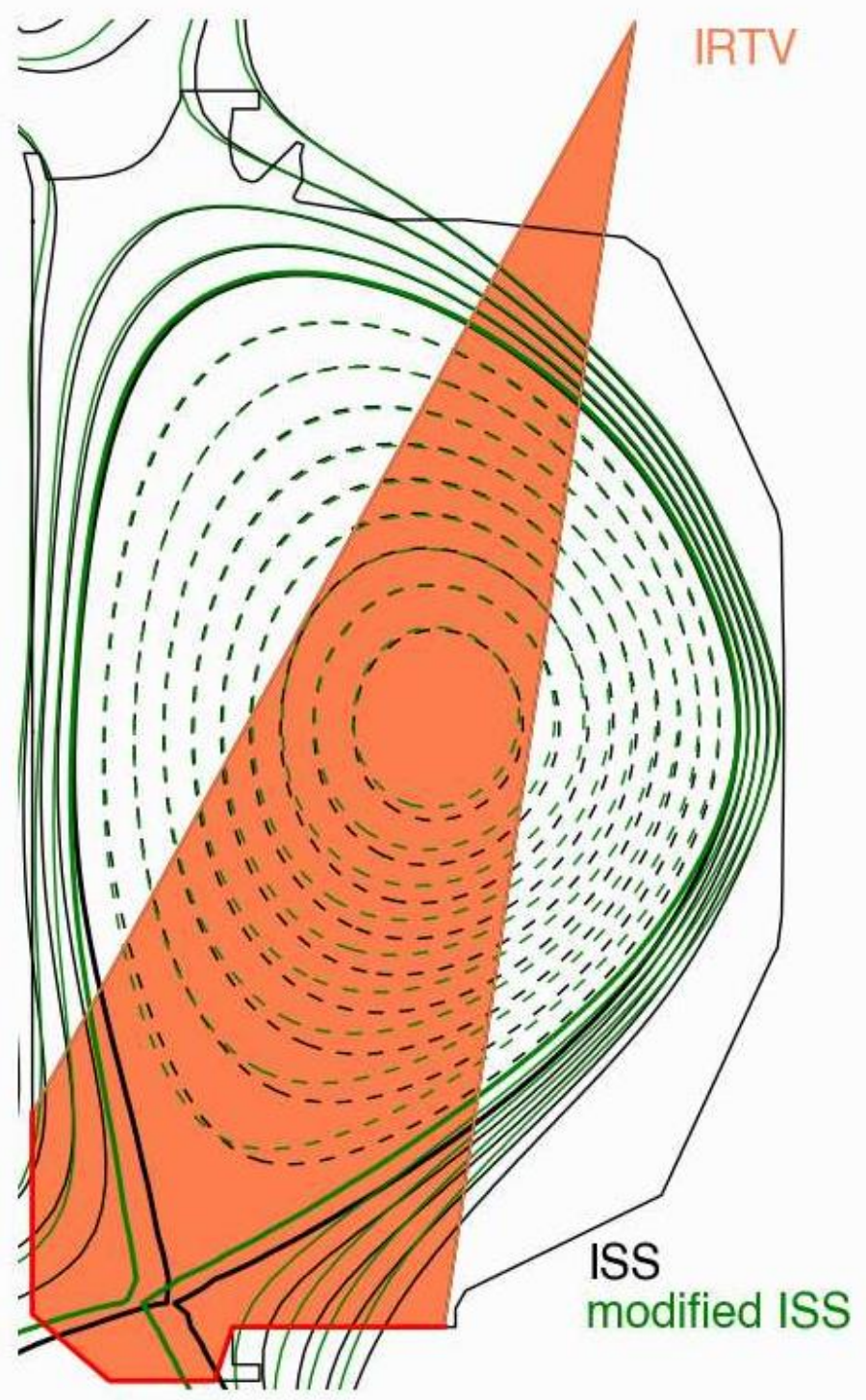

Figure 1 - ITER Similar Shape (ISS) and modified shape for monitoring both strike points with the DIII-D fast IR camera. IR covered range shown in red. 


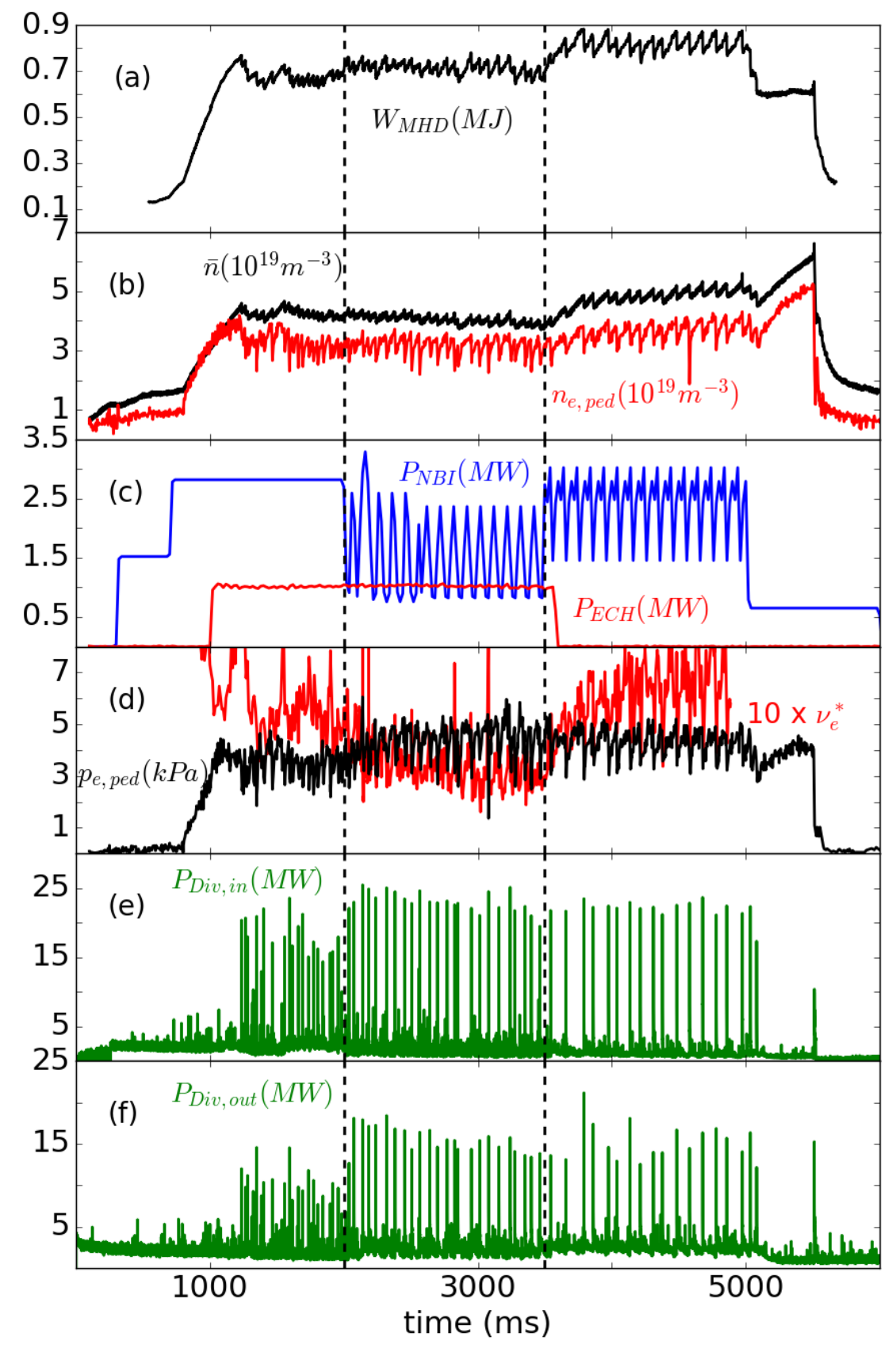

Figure 2 - Evolution of discharge 169430 (1.6 T, $1.12 \mathrm{MA}$ ) with: a) stored MHD energy of the plasma, b) line-averaged (black) and pedestal electron density (red) c) injected power with NBI (blue) and $\mathrm{ECH}$ (red), d) pedestal pressure and electron collisionality at the pedestal (multiplied by a factor of 10), e) inner and f) outer divertor power. The density pump-out effect becomes visible after the ECH turn-off at $3.5 \mathrm{~s}$. 


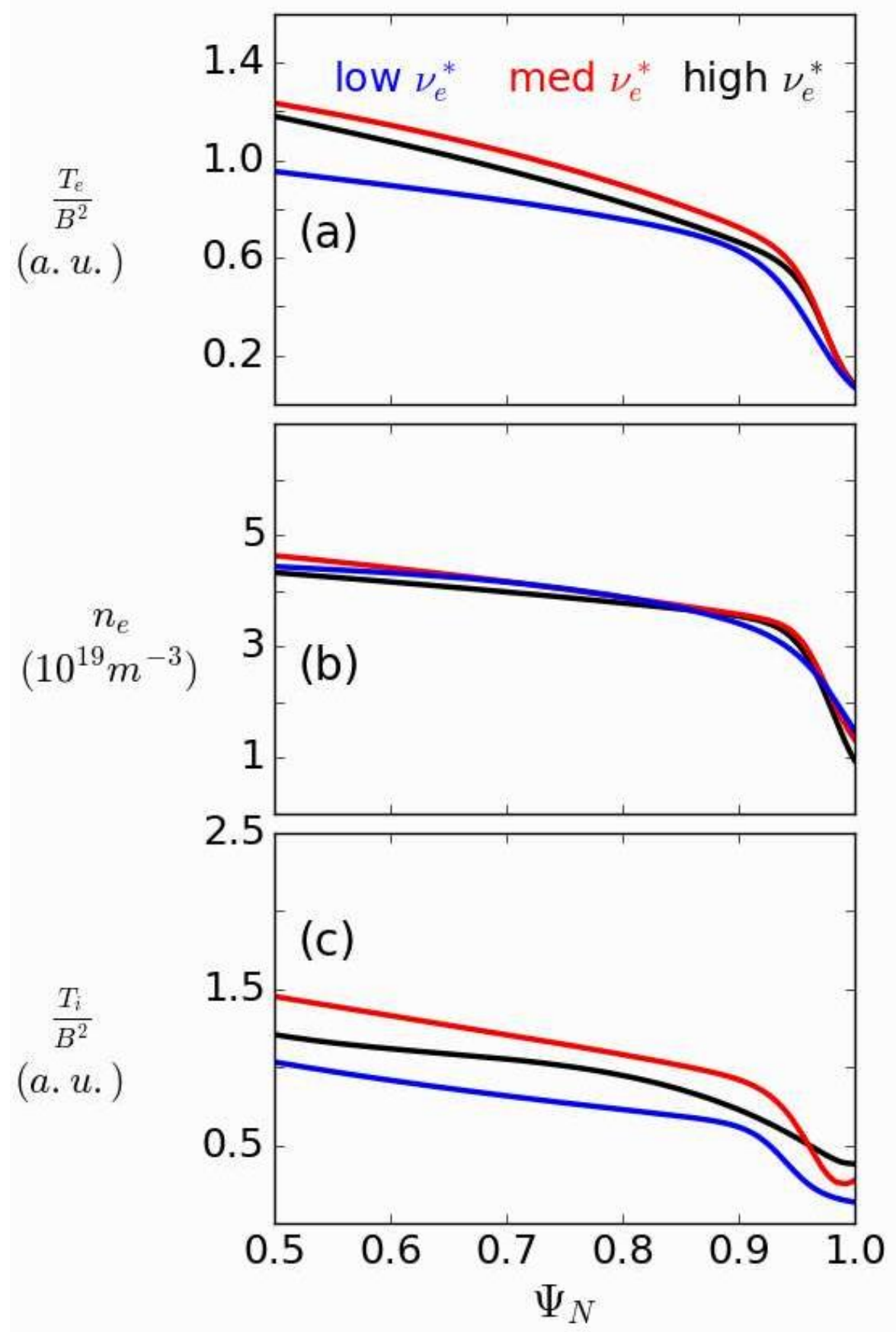

Figure 3 - Profile overview in the outer plasma for the three-point non-dimensional scan: a) normalized electron temperature b) electron density and c) normalized lon temperature. While the density match is good, there are discrepancies in the temperature channel: For a better match the low collisionality case (blue) would require stronger heating, which was avoided to obtain lower ELM frequencies for better diagnostic resolution. 

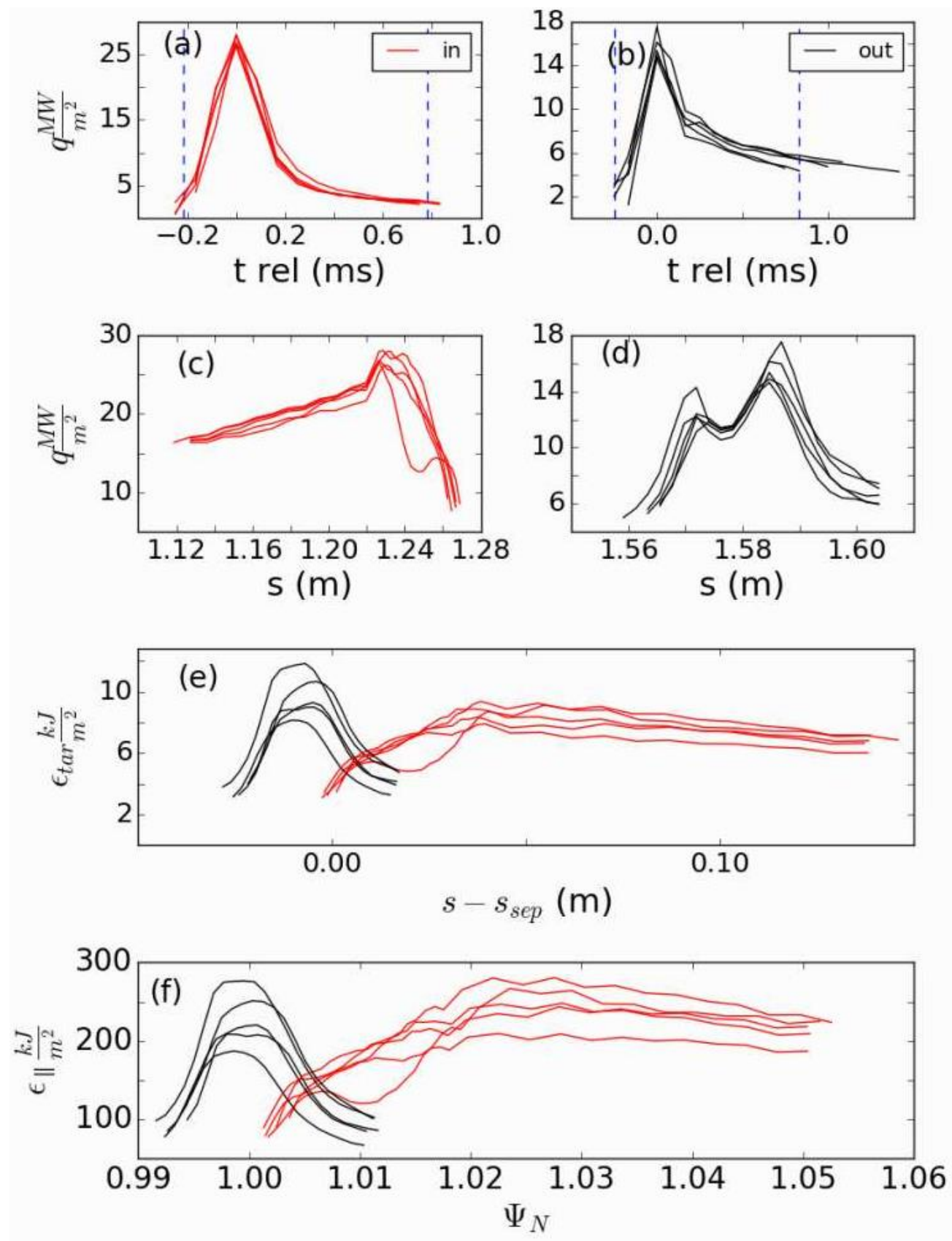

Figure 4-Overview of inner (red) and outer (black) divertor ELM energies during discharge 169426: peak heat flux vs time for inner (a) and outer divertor (b),peak heat flux vs location along IR measurement path for inner (c) and outer (d) divertor, target ELM energy densities relative to separatrix location (e) and parallel ELM energy densities in flux space(f). 


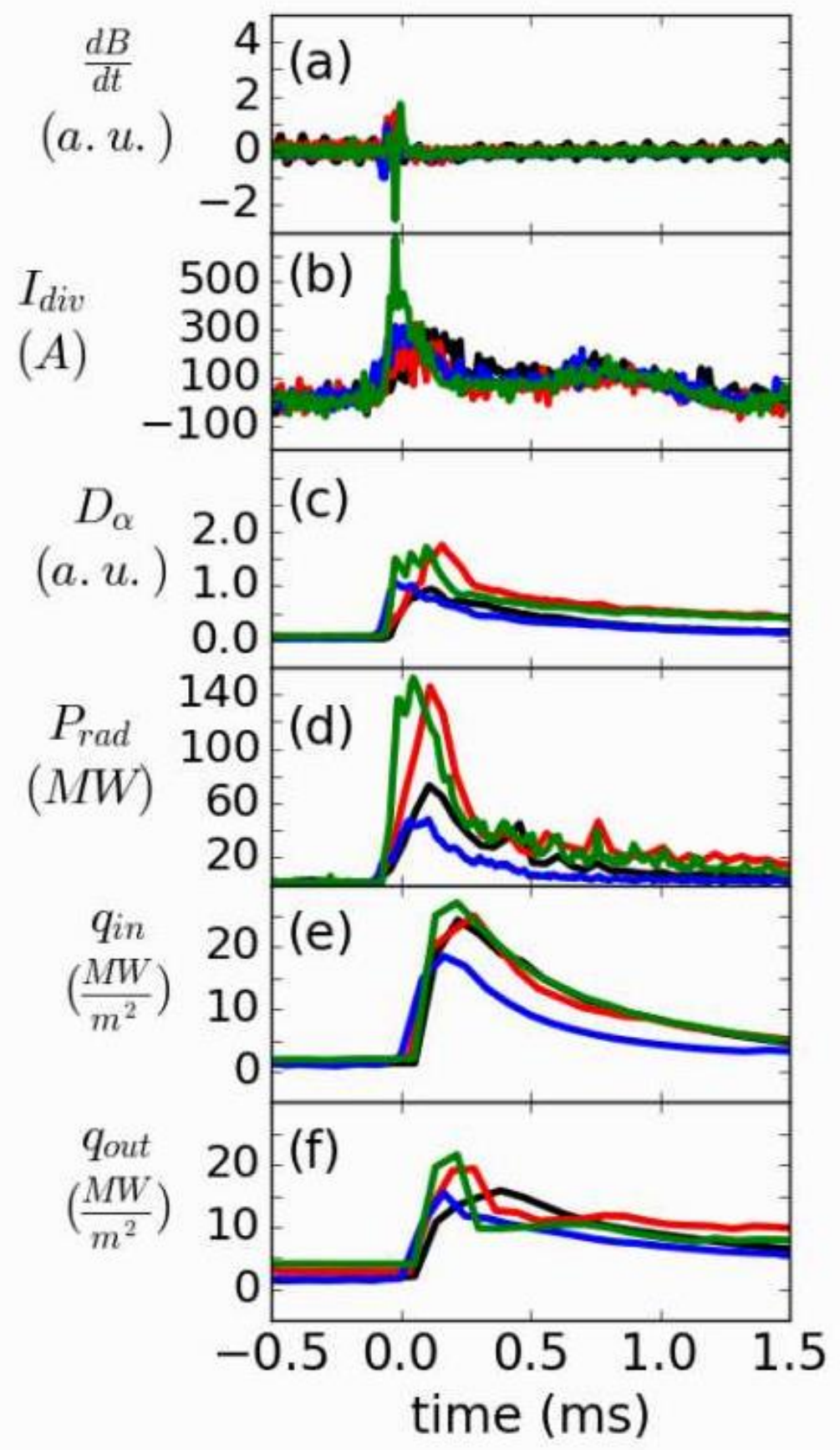

Figure 5 - Time evolution of an ELM in discharges 169431 at $2.4 \mathrm{~s}$ (green), 169432 at $2.7 \mathrm{~s}$ (black), 169433 at $3.0 \mathrm{~s}$ (blue), 169434 at $3.0 \mathrm{~s}$ (red): a) Magnetic midplane toroidal array signal, b) tile current array measurement, c) Dalpha trace, d) radiated power during ELM, e) heat flux towards inner divertor, f) heat flux towards outer divertor. 


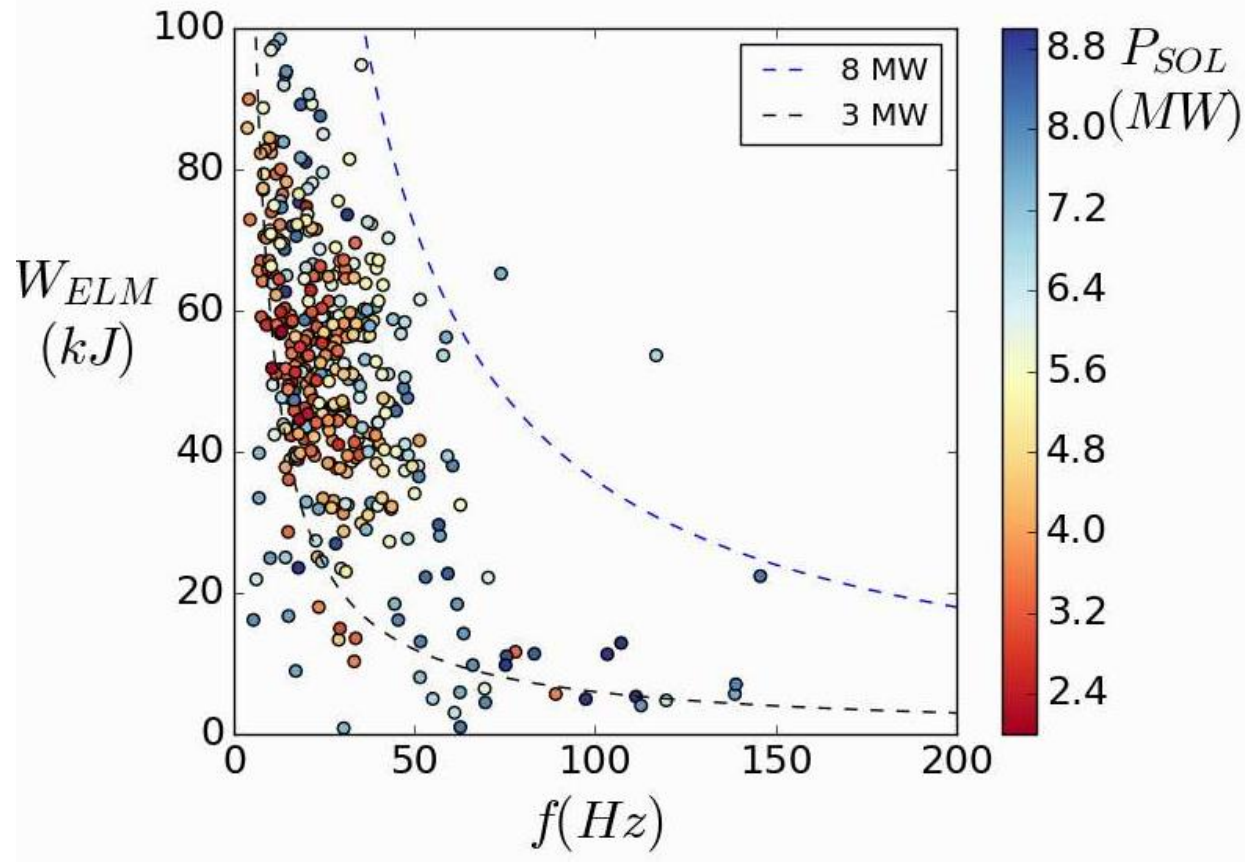

Figure 6-Overview of type-I ELM energy losses $W_{E L M}$ and their dependence on ELM frequency $f$ and power in the scrape-off-layer $P_{S O L}$. Prediction of upper and lower limit for ELM dissipated power during the experiment as explained in text. 


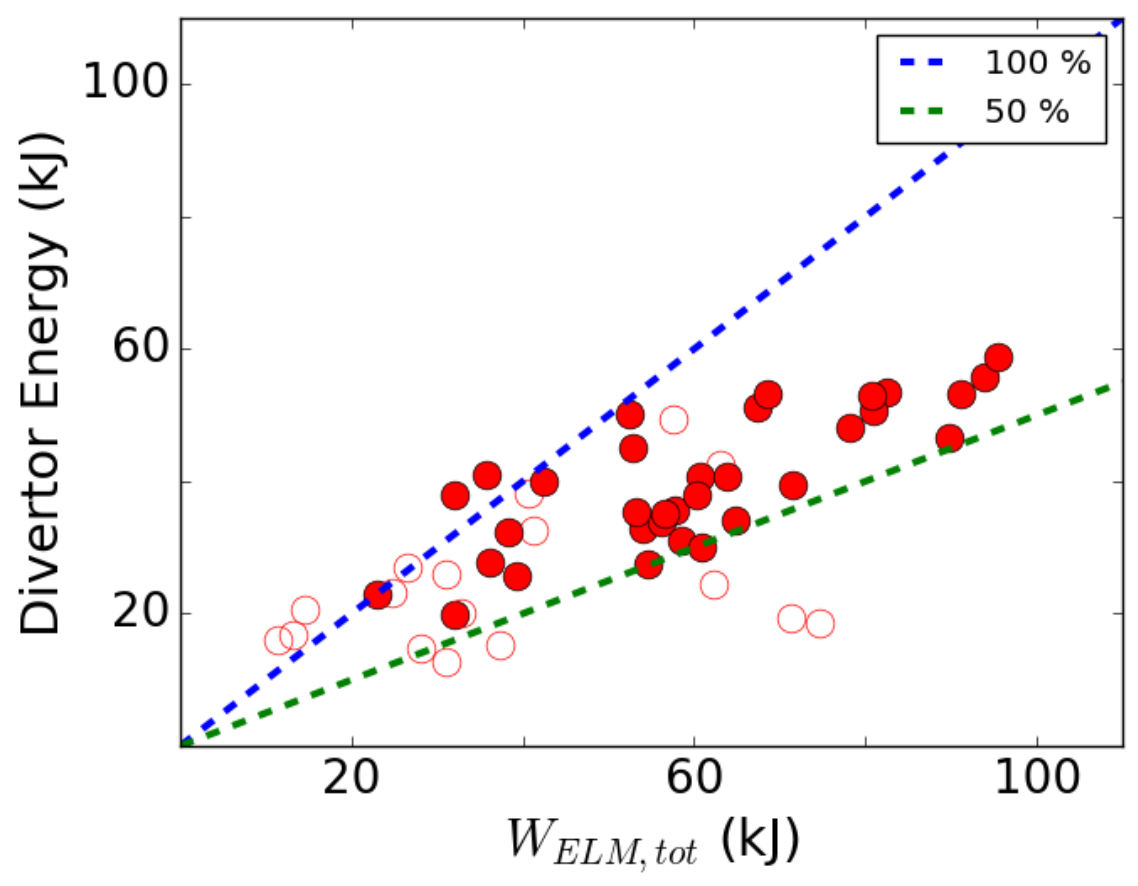

Figure 7 -ELM loss energy in kJ vs ELM energy measured in the divertor for all time intervals considered in the experiment. The dashed lines limit the expectancy cone between $50 \%$ (green) and $100 \%$ (blue) of ELM energy arriving in the divertor. Measurements from previous experiments are distinguished by non-filled markers to demonstrate the generality of the dataset. 


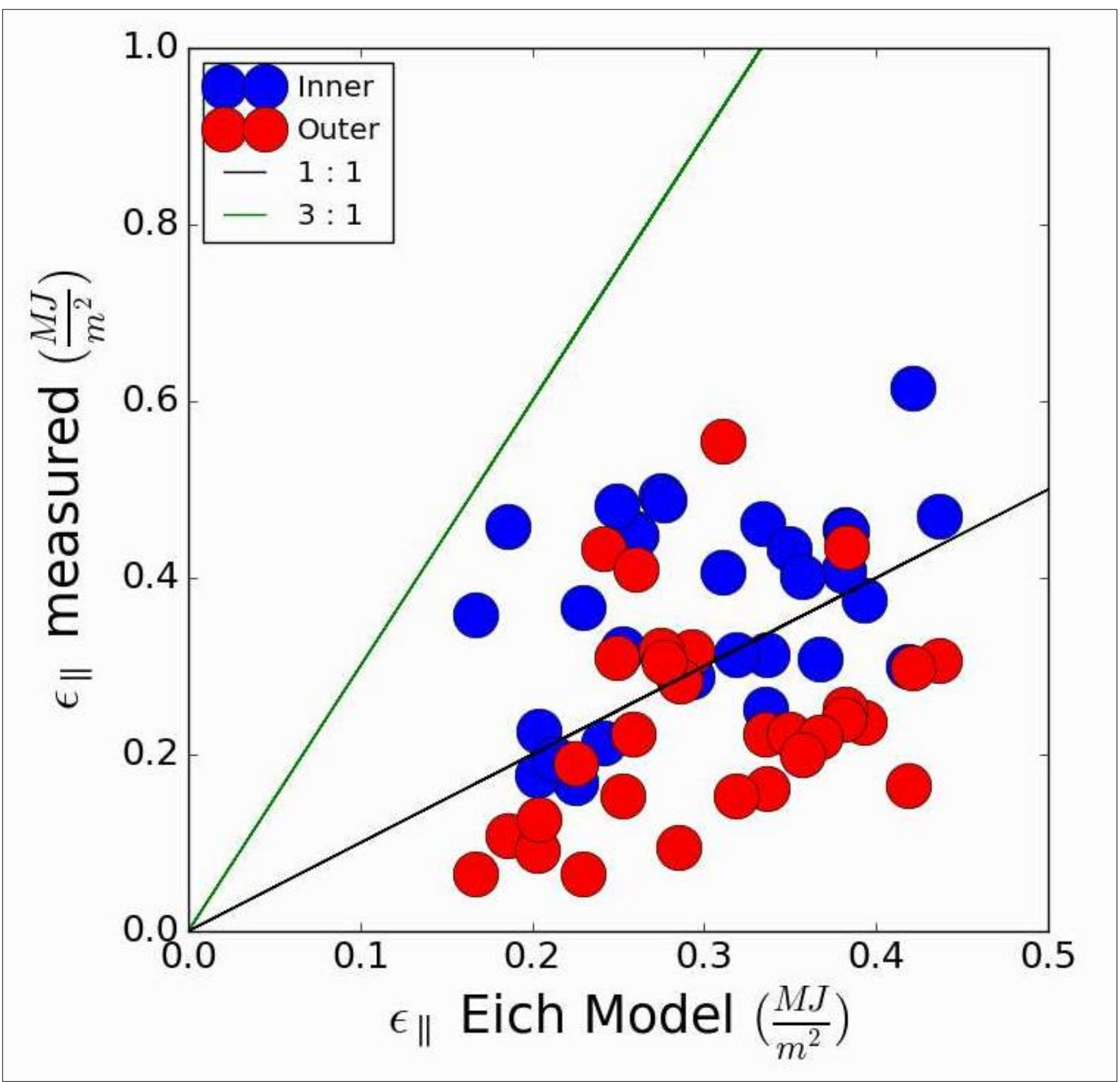

Figure 8 Measured peak parallel ELM energy densities vs Eich Model on DIII-D. 


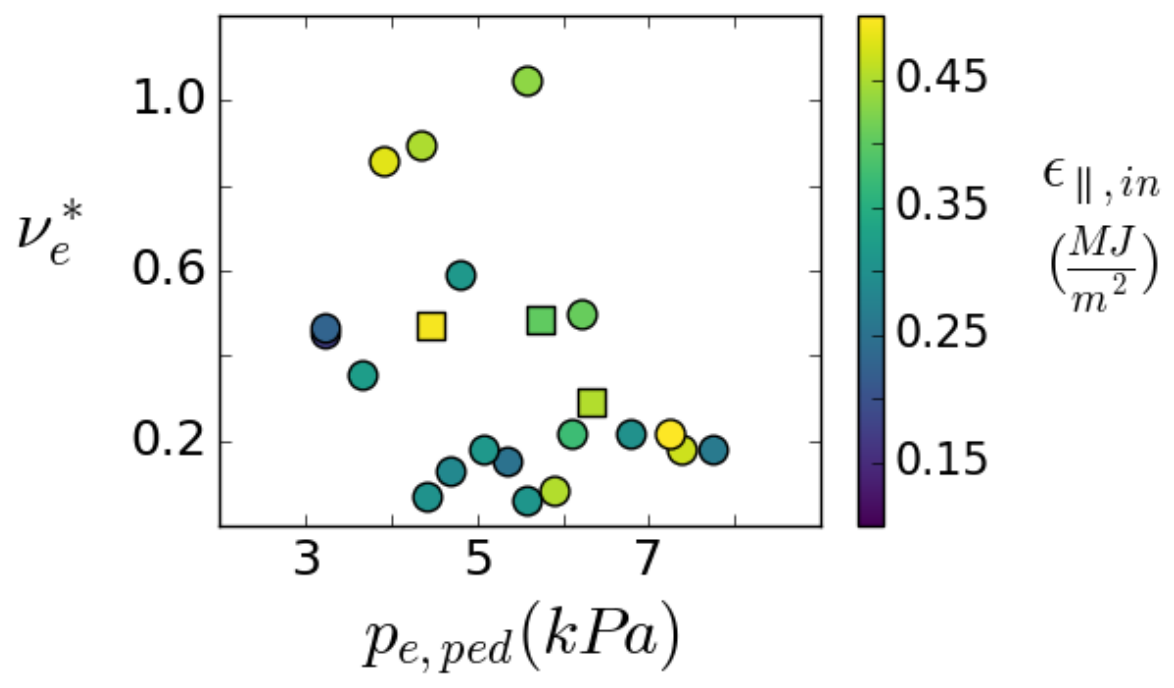

Figure 9 - Dependence of peak parallel ELM energy density to the inner divertor on pedestal electron pressure and pedestal collisonality. The discharges with highlighted profiles in Figure 3 are distinguished by square makers (from left to right: low, high and medium $\mathrm{B}_{\mathrm{T}}$ ) 


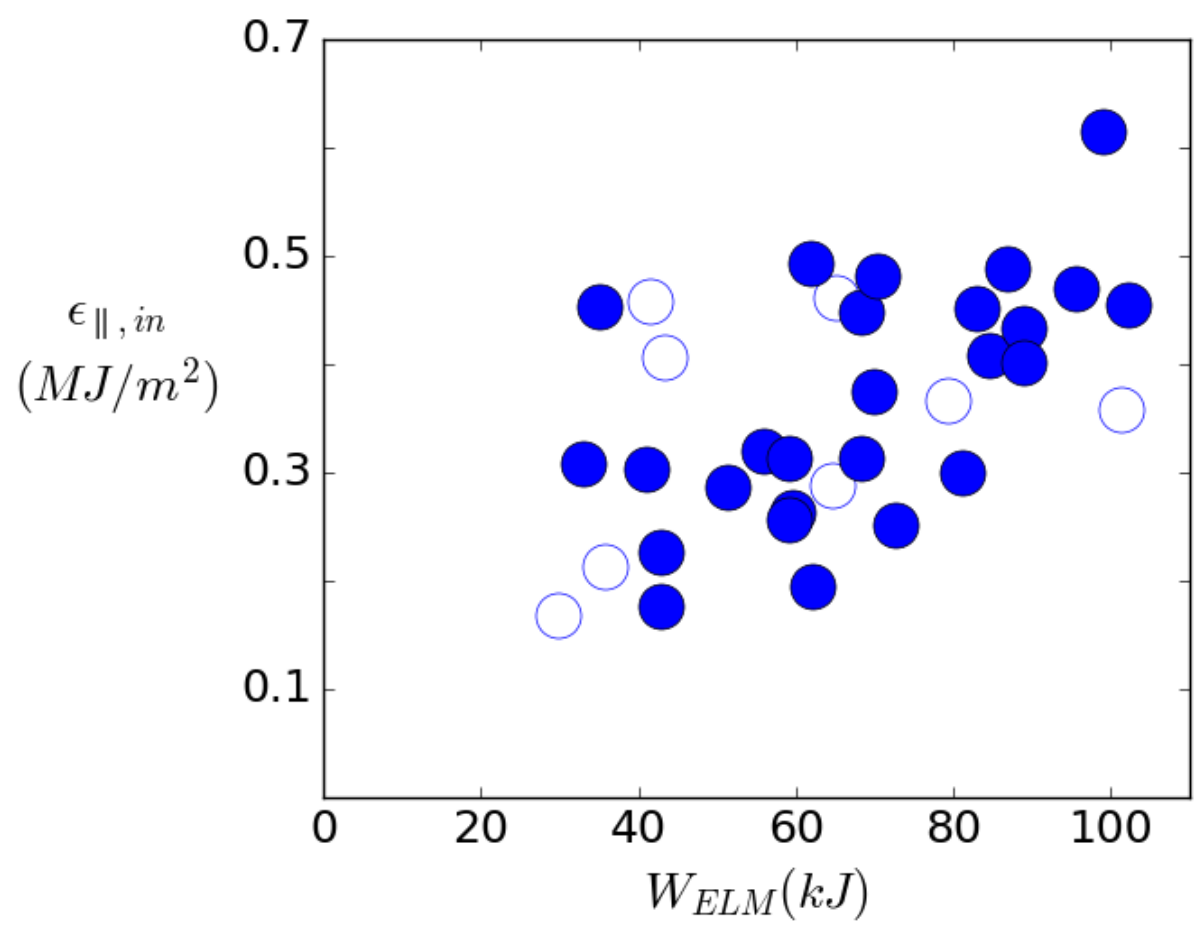

Figure 10 - Dependence of peak parallel ELM energy density to the inner divertor on ELM size. Measurements from previous experiments are distinguished by non-filled markers to demonstrate the generality of the dataset. 

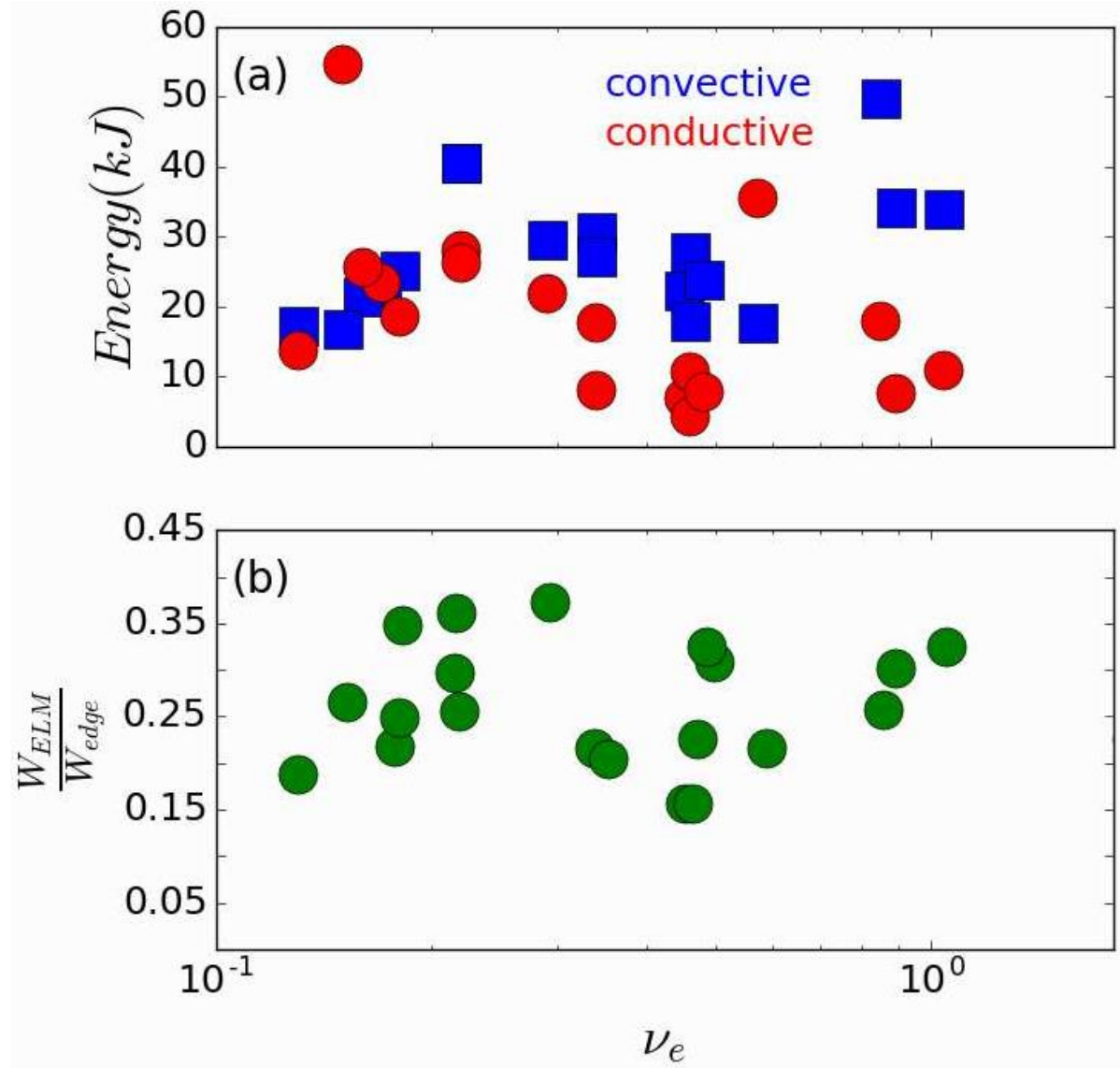

Figure $11-a)$ Conductive and convective losses of the ELM vs. collisionality b) ratio of real ELM loss $W_{E L M}$ to pedestal energy $W_{\text {ped }}$ vs. collisonality 


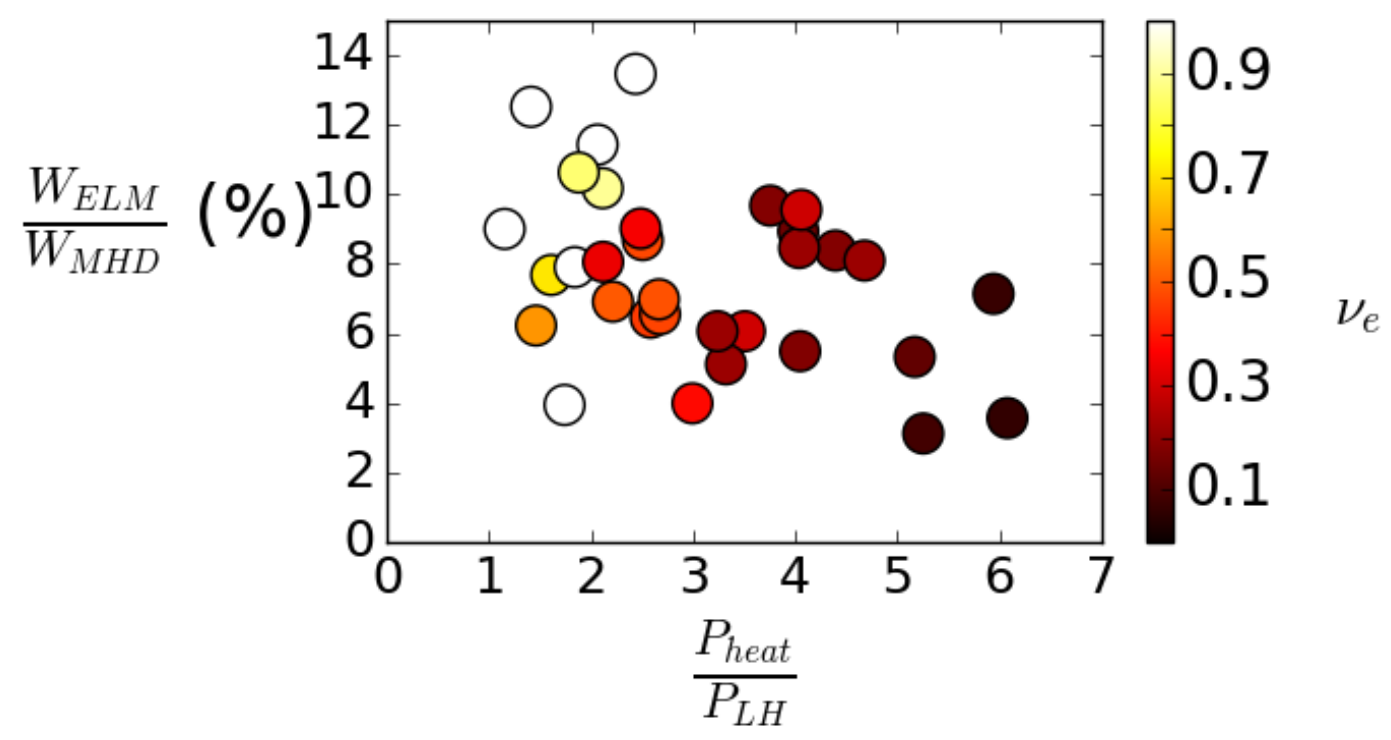

Figure 12 -Relative ELM size in form of fast drop in stored energy $\left(W_{E L M}\right)$ to total stored energy in the plasma $\left(W_{M H D}\right)$ in \% vs relative heating power to $\mathrm{LH}-$ power threshold in dependence of collisionality for all kinetic equilibria of the experiment. 


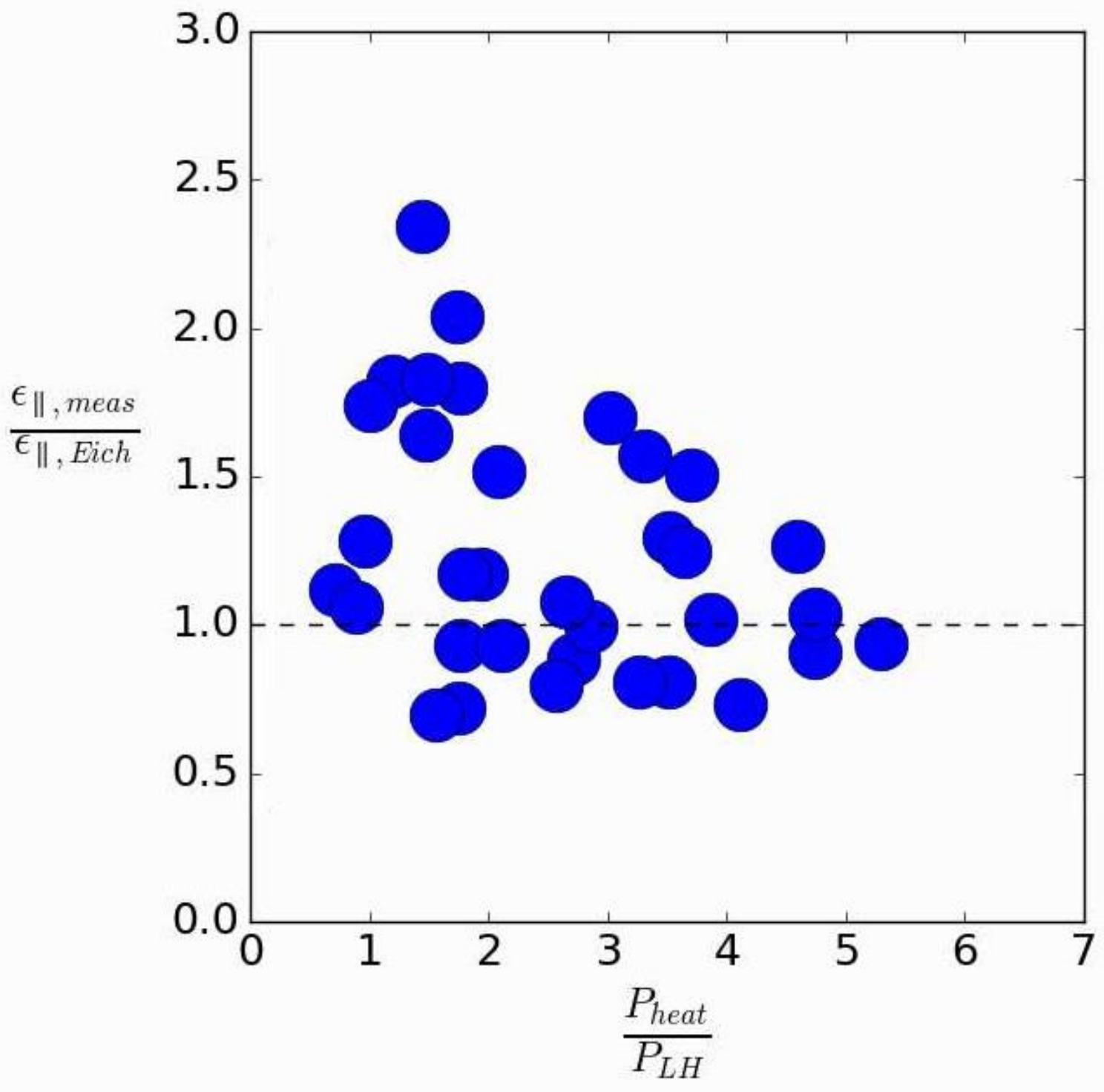

Figure 13 Measured relative ELM energy density to Eich model prediction vs proximity to LHthreshold, regression fit (red) 


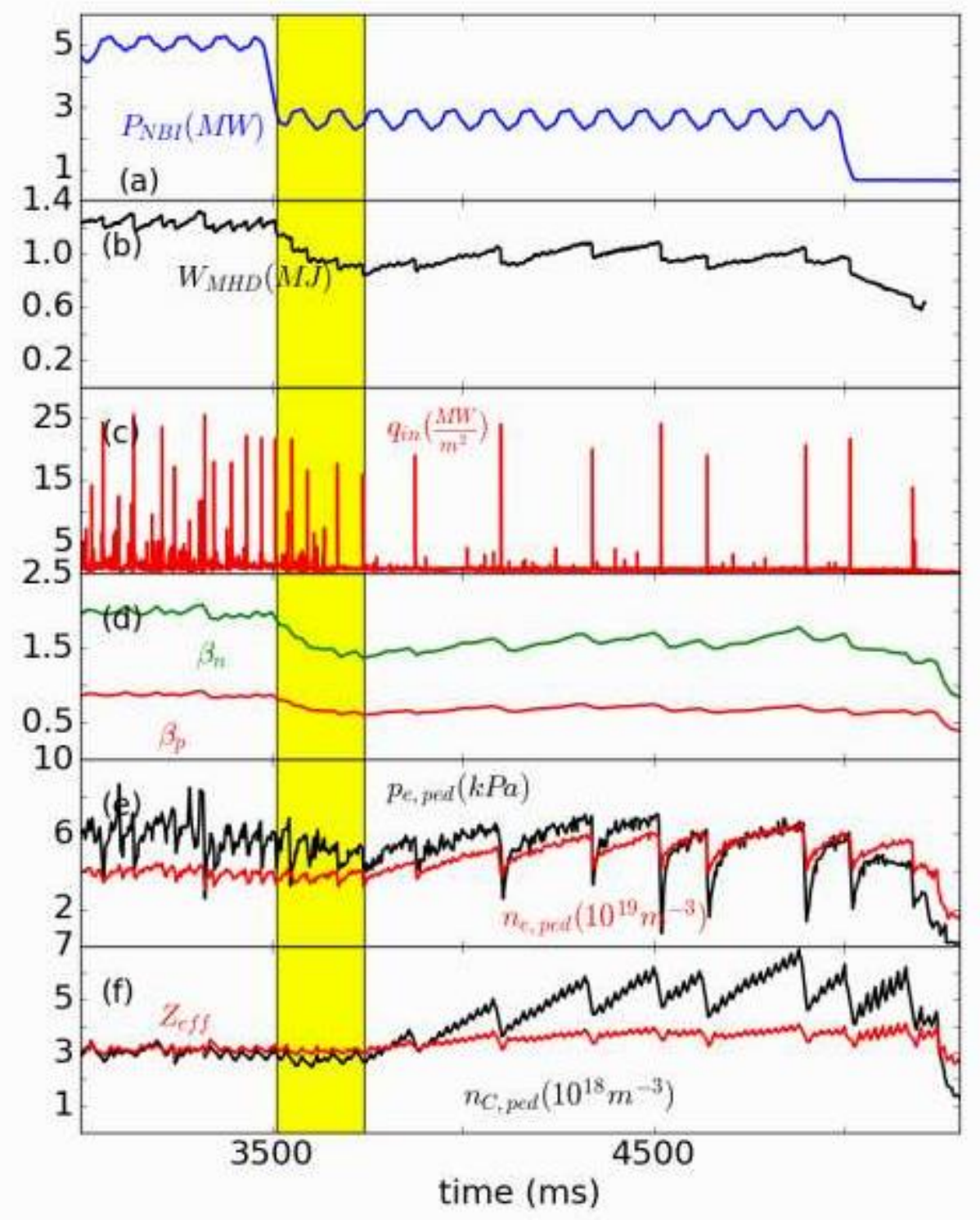

Figure 14 Discharge evolution of 169509 with a power ramp down closer to the LH-threshold: a) injected power with NBI (blue) b) stored MHD energy of the plasma, c) Peak heat flux on inner divertor, d) plasma betas $\beta_{n}$ and $\beta_{p}$ e) pedestal pressure (black) and pedestal electron density (red), f) effective charge and Carbon impurity density at the pedestal top (red), . The yellow window marks the intermediate phase, in which the ELM frequency is still high even though the power has been lowered. After the yellow window big ELMs occur even though the pressure is lower than in the first segment. 


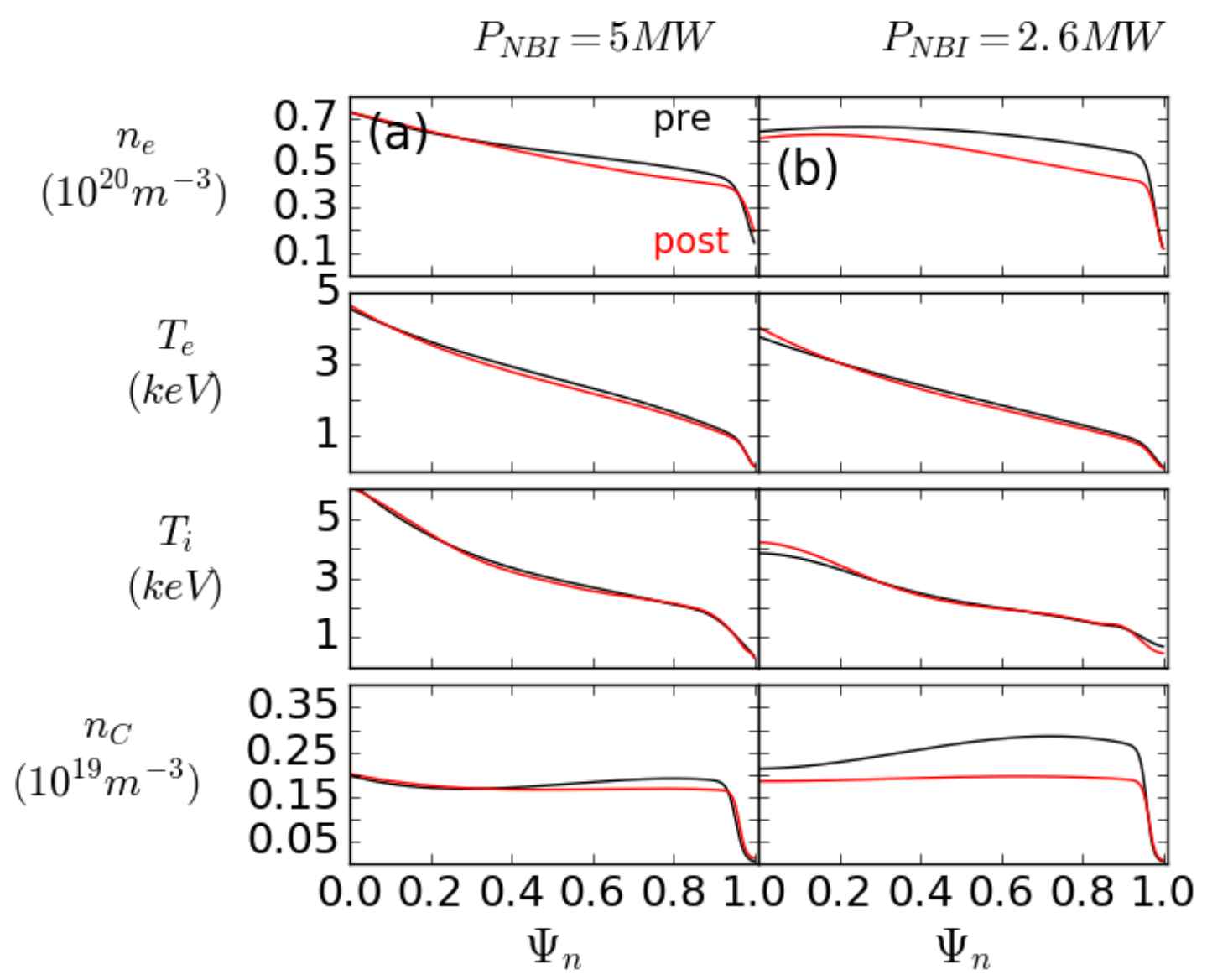

Figure 15 - Comparison of pre (black) and post (red) ELM kinetic profiles for the equilibrium further away $\left(P_{N B}=5 \mathrm{MW}, P_{\text {heat }} / P_{L H}=2.2\right)(\mathrm{a})$ and closer to the LH boundary $\left(P_{N B}=2.6 \mathrm{MW}\right.$, $P_{\text {heat }} / P_{L H}=1.2$ ) (b) and. The traces shown are pedestal electron density and temperature, as well as ion impurity temperature and impurity density. 


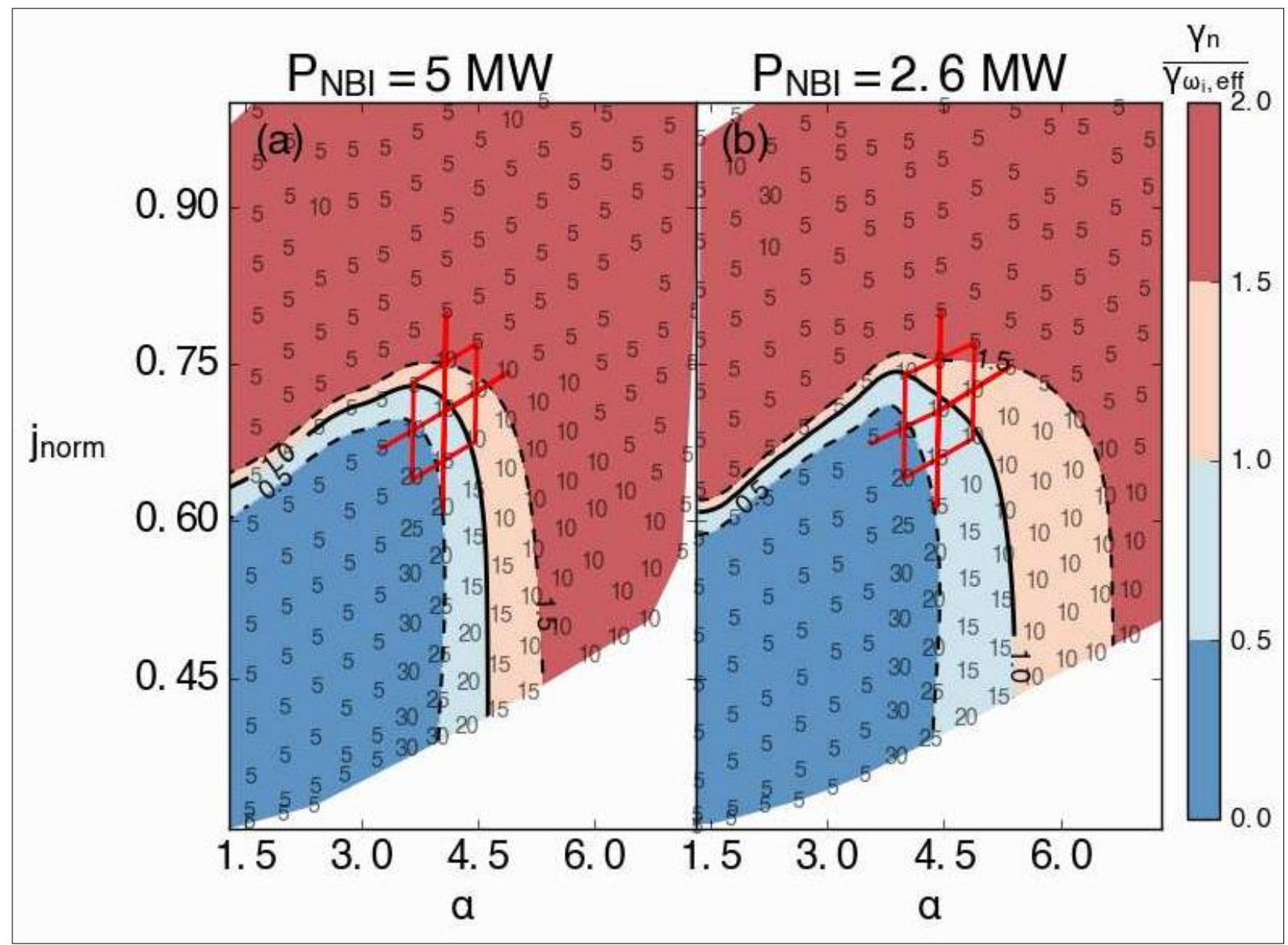

Figure 16 - Comparison of linear stability analysis results before the ELM crashes for discharge 169509: an equilibrium further away from the LH power threshold at $3.0 \mathrm{~s}(\mathrm{a})$ contrasted with a scenario close to $\mathrm{LH}$ threshold at $4.2 \mathrm{~s}(\mathrm{~b})$. The numbers in the plot indicate the most unstable linear mode for each equilibrium, with normalized growth rates $r=\frac{\gamma_{n}}{\gamma_{\omega i, e f f}}$ (to its effective stabilization growth rate) divided in four groups: minimal $(r<0.5$, dark blue), marginal $(0.5<r<1$, light blue), unstable $(1<r<1.5$, yellow), very unstable $(r>1.5$, red). 


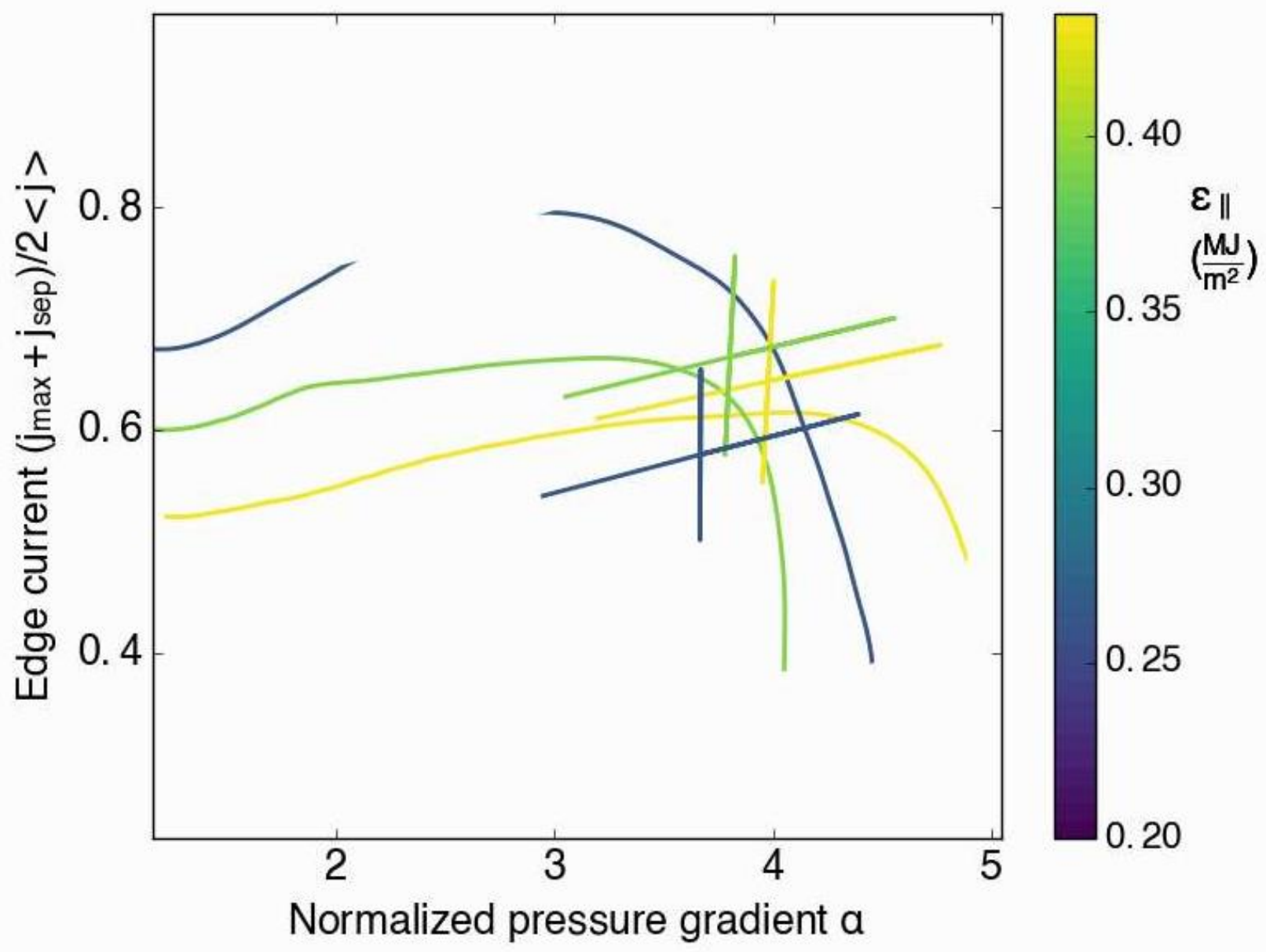

Figure 17 Linear stability of selected equilibria for discharge 153827 (yellow, $n=14$ ), 153828 (green, $n=17$ ) and 153830 (blue, $n=35$ ) with different collisionality and mode numbers. The contour lines show the stability threshold for the respective equilibrium in $\alpha$-j space while the crosses denote the position of the operation point relative to the stability threshold. The peak parallel ELM energy density is indicated by the color of the lines and crosses, with the largest value corresponding to $0.43 \mathrm{MJ} / \mathrm{m}^{2}$ and a most unstable mode number $\mathrm{n}=14$. 


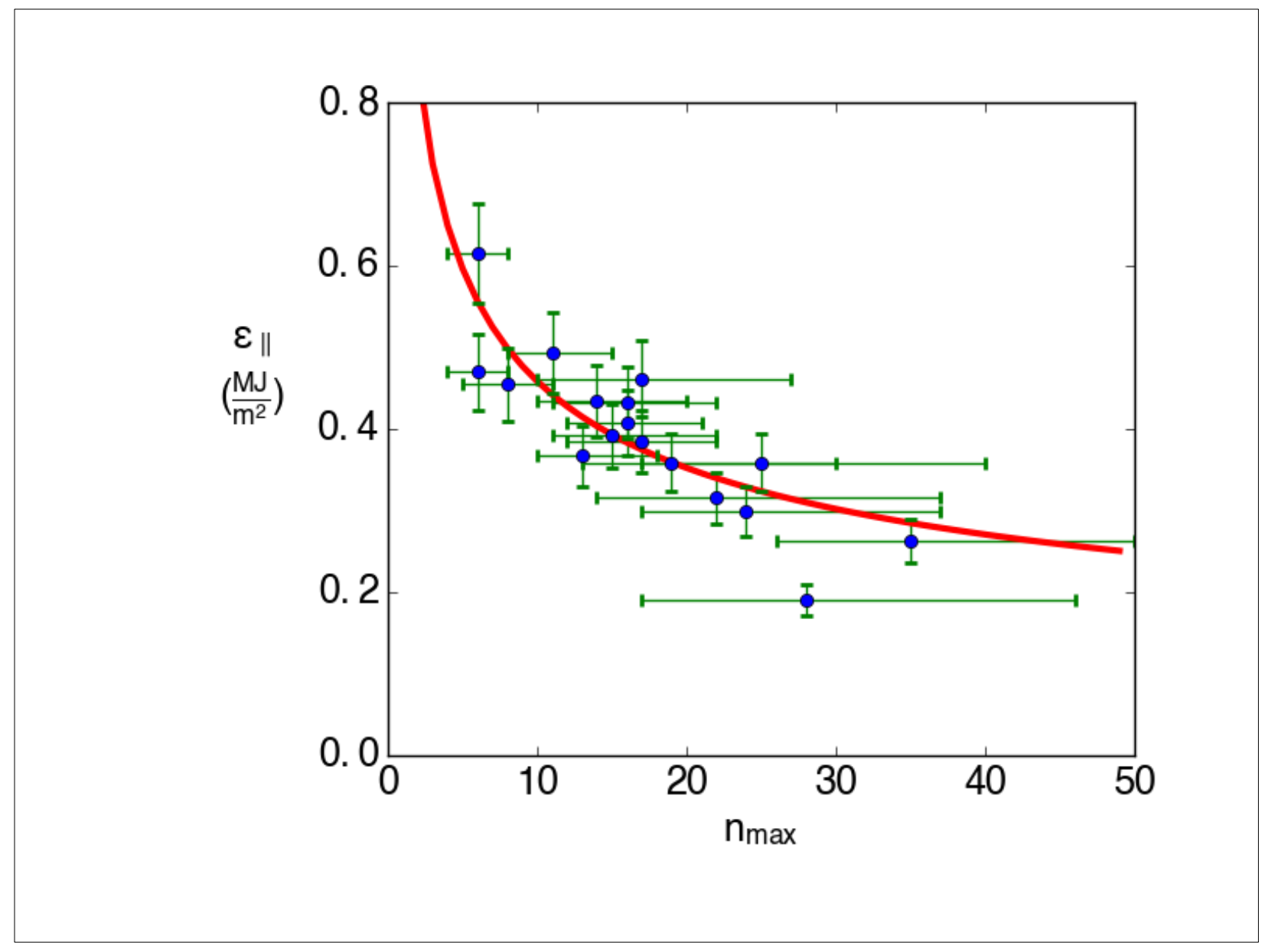

Figure $18 \varepsilon_{\|}$vs. most unstable mode number $n_{\max }$ for a selection of 17 equilibria. The error bar in the abscissa direction stretches over mode numbers with growth rates above $90 \%$ of the $n_{\max }$ growth rate. A $1.1 \cdot n_{\max }{ }^{-0.38}$ fit is included for reference. 


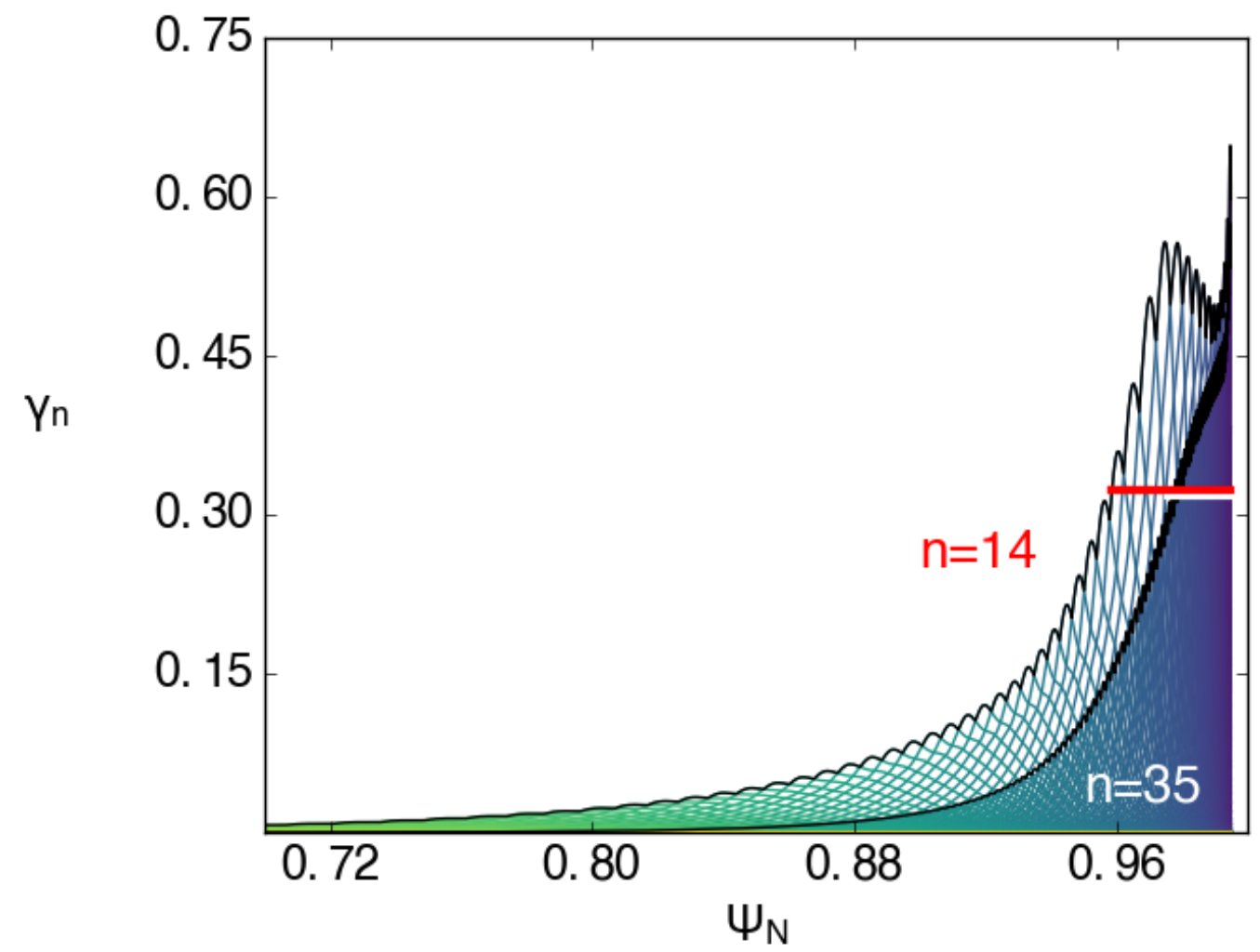

Figure 19 Radial Eigenfunction of most unstable modes: (a) $n=14$ for low collisionality discharge 153827, (b) $n=35$ for high collisionality discharge 153830. As the stability calculation is linear the amplitude is arbitrary. However, the width at half maximum can be used as indicator for the mode penetration. For the lower $\mathrm{n}$ mode number case (red horizontal line) the penetration is almost double that for the $n=35$ case (white horizontal line) and the mode mesh considerably finer. 


\section{REFERENCES}

[1] Eich T, Sieglin B, Thornton A J, Faitsch M, Kirk A, Herrmann A and Suttrop W 2017 ELM divertor peak energy fluence scaling to ITER with data from JET, MAST and ASDEX upgrade Nucl. Mater. Energy 12 84-90

[2] Meneghini O, Smith S P, Lao L L, Izacard O, Ren Q, Park J M, Candy J, Wang Z, Luna C J, Izzo V A, Grierson B A, Snyder P B, Holland C, Penna J, Lu G, Raum P, McCubbin A, Orlov D M, Belli E A, Ferraro N M, Prater R, Osborne T H, Turnbull A D and Staebler G M 2015 Integrated modeling applications for tokamak experiments with OMFIT Nucl. Fusion $\mathbf{5 5}$

[3] Pütterich T, Dux R, Janzer M A and McDermott R M 2011 ELM flushing and impurity transport in the $\mathrm{H}$-mode edge barrier in ASDEX Upgrade Journal of Nuclear Materials vol 415

[4] Linke J, Loewenhoff T, Massaut V, Pintsuk G, Ritz G, Rödig M, Schmidt A, Thomser C, Uytdenhouwen I, Vasechko V and Wirtz M 2011 Performance of different tungsten grades under transient thermal loads Nucl. Fusion $\mathbf{5 1}$

[5] Loarte A, Huijsmans G, Futatani S, Baylor L R, Evans T E, Orlov D M, Schmitz O, Becoulet M, Cahyna P, Gribov Y, Kavin A, Sashala Naik A, Campbell D J, Casper T, Daly E, Frerichs H, Kischner A, Laengner R, Lisgo S, Pitts R A, Saibene G and Wingen A 2014 Progress on the application of ELM control schemes to ITER scenarios from the non-active phase to DT operation Nucl. Fusion 54

[6] Loewenhoff T, Bürger A, Linke J, Pintsuk G, Schmidt A, Singheiser L and Thomser C 2011 Evolution of tungsten degradation under combined high cycle edge-localized mode and steady-state heat loads Physica Scripta T vol T145

[7] Burrell K H, Garofalo A M, Solomon W M, Fenstermacher M E, Osborne T H, Park J-K, Schaffer M J and Snyder P B 2012 Reactor-relevant quiescent H-mode operation using torque from non-axisymmetric, non-resonant magnetic fields Phys. Plasmas 19056117

[8] Evans T E, Moyer R A, Thomas P R, Watkins J G, Osborne T H, Boedo J A, Doyle E J, Fenstermacher M E, Finken K H, Groebner R J, Groth M, Harris J H, La Haye R J, Lasnier C J, Masuzaki S, Ohyabu N, Pretty D G, Rhodes T L, Reimerdes H, Rudakov D L, Schaffer M J, Wang $G$ and Zeng $L 2004$ Suppression of large edge-localized modes in high-confinement DIII-D plasmas with a stochastic magnetic boundary Phys. Rev. Lett. 92

[9] Lang P T, Conway G D, Eich T, Fattorini L, Gruber O, Günter S, Horton L D, Kalvin S, Kallenbach A, Kaufmann M, Kocsis G, Lorenz A, Manso M E, Maraschek M, Mertens V, Neuhauser J, Nunes I, Schneider W, Suttrop W and Urano H 2004 ELM pace making and mitigation by pellet injection in ASDEX upgrade Nucl. Fusion 44 665-77

[10] Leonard A W 2014 Edge-localized-modes in tokamaks Phys. Plasmas 21

[11] Gunn J P, Carpentier-Chouchana S, Dejarnac R, Escourbiac F, Hirai T, Komm M, 
Kukushkin A, Panayotis S and Pitts R A 2017 lon orbit modelling of ELM heat loads on ITER divertor vertical targets Nucl. Mater. Energy 12 75-83

[12] Loarte A, Saibene G, Sartori R, Campbell D, Becoulet M, Horten L, Eich T, Herrmann A, Matthews G, Asakura N, Chankin A, Leonard A, Porter G, Federici G, Janeschitz G, Shimada M and Sugihara M 2003 Characteristics of type I ELM energy and particle losses in existing devices and their extrapolation to ITER Plasma Phys. Control. Fusion 45 154969

[13] Loarte A, Lipschultz B, Kukushkin A S, Matthews G F, Stangeby P C, Asakura N, Counsell G F, Federici G, Kallenbach A, Krieger K, Mahdavi A, Philipps V, Reiter D, Roth J, Strachan J, Whyte D, Doerner R, Eich T, Fundamenski W, Herrmann A, Fenstermacher M, Ghendrih P, Groth M, Kirschner A, Konoshima S, Labombard B, Lang P, Leonard A W, MonierGarbet P, Neu R, Pacher H, Pegourie B, Pitts R A, Takamura S, Terry J and Tsitrone E 2007 Chapter 4: Power and particle control Nucl. Fusion 47

[14] Zohm H 1996 Edge localized modes (ELMs) Plasma Phys. Control. Fusion 38 105-28

[15] Leonard A W, Groebner R J, Mahdavi M A, Osborne T H, Fenstermacher M E, Lasnier C J and Petrie T W 2002 ELM energy scaling in DIII-D Plasma Physics and Controlled Fusion vol 44 pp 945-54

[16] Pitts R A, Andrew P, Arnoux G, Eich T, Fundamenski W, Huber A, Silva C and Tskhakaya D 2007 ELM transport in the JET scrape-off layer Nucl. Fusion 47 1437-48

[17] Eich T, Andrew P, Herrmann A, Fundamenski W, Loarte A and Pitts R A 2007 ELM resolved energy distribution studies in the JET MKII Gas-Box divertor using infra-red thermography Plasma Phys. Control. Fusion 49 573-604

[18] Eich T, Sieglin B, Scarabosio A, Fundamenski W, Goldston R J and Herrmann A 2011 InterELM power decay length for JET and ASDEX Upgrade: Measurement and comparison with heuristic drift-based model Phys. Rev. Lett. 107

[19] Kukushkin A S, Pacher H D, Pacher G W, Janeschitz G, Coster D, Loarte A and Reiter D 2003 Scaling laws for edge plasma parameters in ITER from two-dimensional edge modelling Nucl. Fusion 43 716-23

[20] Pitts R A, Carpentier S, Escourbiac F, Hirai T, Komarov V, Kukushkin A S, Lisgo S, Loarte A, Merola M, Mitteau R, Raffray A R, Shimada M and Stangeby P C 2011 Physics basis and design of the ITER plasma-facing components Journal of Nuclear Materials vol 415

[21] Sauter O, Angioni C and Lin-Liu Y R 1999 Neoclassical conductivity and bootstrap current formulas for general axisymmetric equilibria and arbitrary collisionality regime Phys.

Plasmas 6 2834-9

[22] Petty C C, Luce T C, Burrell K H, Chiu S C, DeGrassie J S, Forest C B, Gohil P, Greenfield C M, Groebner R J, Harvey R W, Pinsker R I, Prater R, Waltz R E, James R A and Wròblewski 
D 1995 Nondimensional transport scaling in DIII-D: Bohm versus gyro-Bohm resolved Phys. Plasmas 2 2342-8

[23] Hill D N, Ellis R, Ferguson W, Perkins D E, Petrie T and Baxi C 1988 Infrared thermography of the DIII-D divertor targets Rev. Sci. Instrum. 59 1878-80

[24] Herrmann A, Junker W, Gunther K, Bosch S, Kaufmann M, Neuhauser J, Pautasso G, Richter T and Schneider R 1995 Energy flux to the ASDEX-Upgrade diverter plates determined by thermography and calorimetry Plasma Phys. Control. Fusion 37 17-29

[25] Mc Carthy P J, Horton L, Kallenbach A and Kurzan B 2003 ELM-resolved interpretive MHD equilibria on ASDEX Upgrade using SOL tile currents and kinetic data St. Petersbg. 27 7-11

[26] Hollmann E M, Chousal L, Fisher R K, Hernandez R, Jackson G L, Lanctot M J, Pidcoe S V., Shankara J and Taussig D A 2011 Soft x-ray array system with variable filters for the DIII-D tokamak Rev. Sci. Instrum. 82

[27] Evans T E, Lasnier C J, Hill D N, Leonard A W, Fenstermacher M E, Petrie T W and Schaffer M J 1995 Measurements of non-axisymmetric effects in the DIII-D divertor J. Nucl. Mater. 220-222 235-9

[28] Lao L L, John H S, Stambaugh R D, Kellman A G and Pfeiffer W 1985 Reconstruction of current profile parameters and plasma shapes in tokamaks Nucl. Fusion 25 1611-22

[29] Groebner R J, Baker D R, Burrell K H, Carlstrom T N, Ferron J R, Gohil P, Lao L L, Osborne $T H$, Thomas D M, West W P, Boedo J A, Moyer R A, McKee G R, Deranian R D, Doyle E J, Rettig C L, Rhodes T L and Rost J C 2001 Progress in quantifying the edge physics of the $H$ mode regime in DIII-D Nucl. Fusion 41 1789-802

[30] Herrmann A 2002 Overview on stationary and transient divertor heat loads Plasma Physics and Controlled Fusion vol 44 pp 883-903

[31] Leonard A W, Mahdavi M A, Lasnier C J, Petrie T W and Stangeby P C 2012 Scaling radiative divertor solutions to high power in DIII-D Nucl. Fusion $\mathbf{5 2}$

[32] Bortolon A, Maingi R, Mansfield D K, Nagy A, Roquemore A L, Baylor L R, Commaux N, Jackson G L, Gilson E P, Lunsford R, Parks P B, Chrystal C, Grierson B A, Groebner R, Haskey S R, Makowski M J, Lasnier C J, Nazikian R, Osborne T, Shiraki D and Van Zeeland M A 2016 High frequency pacing of edge localized modes by injection of lithium granules in DIII-D H-mode discharges Nucl. Fusion 56

[33] Taylor T S, St. John H, Turnbull A D, Lin-Liu V R, Burrell K H, Chan V, Chu M S, Ferron J R, Lao L L, La Haye R J, Lazarus E A, Miller R L, Politzer P A, Schissel D P and Strait E J 1994 Optimized profiles for improved confinement and stability in the DIII-D tokamak Plasma Phys. Control. Fusion 36

[34] Hawryluk R J 1981 AN EMPIRICAL APPROACH TO TOKAMAK TRANSPORT Physics of 
Plasmas Close to Thermonuclear Conditions pp 19-46

[35] Pankin A, McCune D, Andre R, Bateman G and Kritz A 2004 The tokamak Monte Carlo fast ion module NUBEAM in the national transport code collaboration library Comput. Phys. Commun. 159 157-84

[36] Martin Y R and Takizuka T 2008 Power requirement for accessing the H-mode in ITER J. Phys. Conf. Ser. 123

[37] Miller R L, Chu M S, Greene J M, Lin-Liu Y R and Waltz R E 1998 Noncircular, finite aspect ratio, local equilibrium model Phys. Plasmas 5 973-8

[38] Snyder P B, Wilson H R, Ferron J R, Lao L L, Leonard A W, Osborne T H, Turnbull A D, Mossessian D, Murakami M and Xu X Q 2002 Edge localized modes and the pedestal: A model based on coupled peeling-ballooning modes Physics of Plasmas vol 9 pp 2037-43

[39] Snyder P B, Osborne T H, Burrell K H, Groebner R J, Leonard A W, Nazikian R, Orlov D M, Schmitz O, Wade M R and Wilson H R 2012 The EPED pedestal model and edge localized mode-suppressed regimes: Studies of quiescent $\mathrm{H}$-mode and development of a model for edge localized mode suppression via resonant magnetic perturbations Phys. Plasmas 19

[40] Liang Y, Koslowski H R, Thomas P R, Nardon E, Alper B, Andrew P, Andrew Y, Arnoux G, Baranov $Y$, Bécoulet $M$, Beurskens $M$, Biewer T, Bigi M, Crombe K, De La Luna E, De Vries P, Fundamenski W, Gerasimov S, Giroud C, Gryaznevich M P, Hawkes N, Hotchin S, Howell D, Jachmich S, Kiptily V, Moreira L, Parail V, Pinches S D, Rachlew E and Zimmermann O 2007 Active control of type-I edge-localized modes with $n=1$ perturbation fields in the JET tokamak Phys. Rev. Lett. 98

[41] Suttrop W, Eich T, Fuchs J C, Günter S, Janzer A, Herrmann A, Kallenbach A, Lang P T, Lunt T, Maraschek M, McDermott R M, Mlynek A, Pütterich T, Rott M, Vierle T, Wolfrum E, Yu Q, Zammuto I and Zohm H 2011 First observation of edge localized modes mitigation with resonant and nonresonant magnetic perturbations in ASDEX upgrade Phys. Rev. Lett. 106

[42] Snyder P B, Wilson H R, Osborne T H and Leonard A W 2004 Characterization of peelingballooning stability limits on the pedestal Plasma Phys. Control. Fusion 46 A131-41

[43] Huijsmans G T A and Loarte A 2013 Non-linear MHD simulation of ELM energy deposition Nucl. Fusion $\mathbf{5 3}$

[44] Pamela S, Eich T, Frassinetti L, Sieglin B, Saarelma S, Huijsmans G, Hoelzl M, Becoulet M, Orain F, Devaux S, Chapman I, Lupelli I, Solano E and Contributors J E T 2015 Non-linear MHD simulations of ELMs in JET and quantitative comparisons to experiments Plasma Phys. Control. Fusion 58

[45] Hayashi N, Takizuka T, Aiba N, Oyama N, Ozeki T, Wiesen S and Parail V 2009 Integrated simulation of ELM energy loss and cycle in improved H-mode plasmas Nucl. Fusion 49 
095015

[46] Hayashi N, Oyama N, Takizuka T, Aiba N and Ozeki T 2011 Effect of core pressure gradient just inside the top of the pedestal on the energy loss due to the edge localized mode in JT-60U Nucl. Fusion $\mathbf{5 1} 073015$

[47] Snyder P B, Groebner R J, Hughes J W, Osborne T H, Beurskens M, Leonard A W, Wilson $H$ R and Xu X Q 2011 A first-principles predictive model of the pedestal height and width: Development, testing and ITER optimization with the EPED model Nucl. Fusion $\mathbf{5 1}$ 